\title{
Trends in Incidence and Mortality of
}

\section{Gynecological and Breast Cancers in Poland (1980-2018)}

\author{
Marcin Piechocki (D)', Wojciech Koziołek (D)', Damian Sroka', Anna Matrejek', Paulina Miziołek', \\ Nazarii Saiuk', Monika Sledzik', Adriana Jaworska', Krzysztof Bereza², Elzbieta Pluta ${ }^{3}$, \\ Tomasz Banas (D)
}

'Department of Gynecology and Oncology, Jagiellonian University Medical College, Krakow, Poland; ${ }^{2}$ Department of Mother and Child Health;Faculty of Health Sciences;Institute of Nursing and Midwifery;jagiellonian University Medical College, Krakow, Poland; ${ }^{3}$ Department of Radiotherapy, Maria Sklodowska-Curie Institute - Oncology Centre, Krakow, Poland

Correspondence: Tomasz Banas, Department of Gynecology and Oncology, Jagiellonian University Medical College, 2nd Jakubowskiego Str, Krakow, Poland, Tel +48 I2 424 8584, Fax +48 I2 424 8560, Email tomasz.I.banas@uj.edu.pl

Background: This study aimed to analyze and determine the incidence and mortality trends in gynecological and breast cancers (BCs) in Poland. The gynecological cancers assessed were cervical cancer (CC), corpus uteri cancer (CUC), ovarian cancer (OC), vaginal cancer (VAC), and vulvar cancer (VUC).

Patients and Methods: Data concerning the incidence and mortality for the period of 1980-2018 were obtained from the Polish National Cancer Registry (PNCR). Joinpoint regression analysis was performed to identify trends, which were described using the annual percentage change (APC) and the average annual percent change (AAPC).

Results: Statistically significant increases were observed in $\mathrm{BC}$ incidence (AAPC: 2.3; $\mathrm{CI}$ : 1.8 to 2.9; $<<0.05$ ), CUC incidence (AAPC: 2.3 ; CI: 1.9 to 2.7 ; $<<0.05$ ), CUC mortality (AAPC: 0.4 ; CI: 0.1 to $0.7 ; \mathrm{p}<0.05$ ) and VUC mortality (AAPC: 1.16 , CI: 0.1 to 2.2; $<<0.05$ ). VAC mortality decreased (AAPC: -3.5 , CI: -5.0 to $-2.0 ; \mathrm{p}<0.05$ ), as did CC incidence and mortality (AAPC: -2.1 , CI: -2.3 to -1.8 ; $<<0.05$, AAPC: $-2.0, \mathrm{CI}:-2.2$ to -1.8 ; $<<0.05$, respectively). Between 1980 and 1993, OC incidence initially increased and then stabilized (AAPC: 0.9 ; CI: 0.7 to $1.1 ; \mathrm{p}<0.05$ ). After 2007 , OC mortality decreased (AAPC: $0.0 ; \mathrm{CI}$ : -0.2 to $0.2 ; \mathrm{p}=0.8$ ). Trends in VUC and VAC incidence and BC mortality were not statistically significant.

Conclusion: The results of this study showed a significant increase in OC, CUC, and BC incidence, and a decrease in the incidence of $\mathrm{CC}$ and VAC. The VUC trends were stable. Mortality trends for BC initially fluctuated and, since 2010, has begun to increase. Throughout the observed period, mortality due to VUC and CUC increased, whereas decreased among patients with CC. OC mortality was stable, but not significant. Furthermore, the study showed a correlation between age group and rate of incidence and mortality of each assessed cancer.

Keywords: gynecological cancers, breast cancer, incidence, mortality, average annual percentage change

\section{Introduction}

Gynecological cancers constitute a serious public health problem because gynecological malignancies continue to be an important cause of cancer-related mortality. With an estimated annual incidence of more than 3.6 million and mortality exceeding 1.3 million, these cancers account for nearly $40 \%$ of all cancer incidence and for more than $30 \%$ of all cancer mortality in women worldwide. ${ }^{1}$ Breast cancer (BC) and corpus uteri cancer (CUC) are the top three malignancies involving the female reproductive system worldwide and are strongly related to lifestyle, socioeconomic conditions, and demographic changes. ${ }^{1}$ Increasing wealth within Polish society during recent years, combined with successive lifestyle changes, has resulted in an increase in Western diseases, such as diabetes, obesity, and hypertension-all of which may be risk factors for some of these malignancies. ${ }^{2,3}$ 


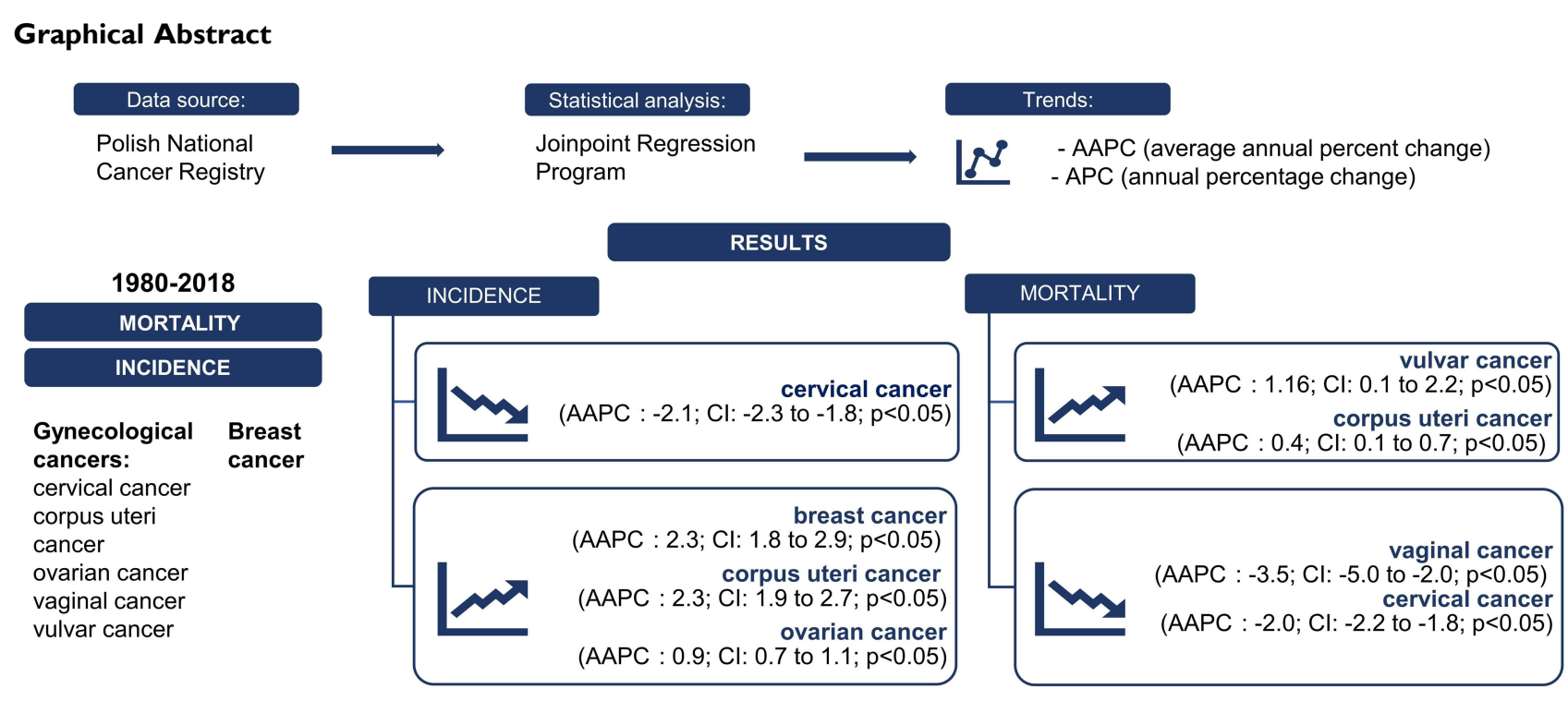

In 2020, BC surpassed lung cancer as the most common type of cancer diagnosed in women, with an estimated 2.3 million new cases in 2020 accounted for $6.9 \%$ of all cancer-related deaths, making it the leading cause of cancer death in women as well. ${ }^{1}$ The most important risk factors for BC are increased estrogen exposure (eg, related to early menarche, late menopause, nulliparity, or hormonal contraception); short duration, or total lack, of breastfeeding; obesity; diabetes; and genetic mutations. ${ }^{4-6}$

Cervical cancer (CC) is currently the second most common gynecological cancer, with 0.6 million new cases in 2020, and it is the fourth leading cause of cancer death in women, with approximately 342,000 deaths worldwide. ${ }^{1}$ Human papillomavirus (HPV), with its 12 oncogenic types, is the major risk factor for $\mathrm{CC} .{ }^{1,7-9} \mathrm{CC}$ is considered nearly completely preventable because of the highly effective primary prevention method (the HPV vaccine) as well as secondary prevention measures. ${ }^{1}$

CUC was the third most diagnosed cancer in women, with 417,000 new cases and 97,000 deaths in $2020 .{ }^{1}$ Incidence rates vary depending on ethnicity and are higher in developed countries, mainly because of increased risk factors (ie, obesity; increased estrogen exposure; infertility, particularly in the presence of polycystic ovarian syndrome; and longterm hormone replacement therapy). ${ }^{1,10,11}$

Ovarian cancer (OC) is the fourth most common gynecological malignancy, with 313,959 new cases and 207,252 deaths in 2020, making it the most lethal gynecological cancer. ${ }^{1,12,13}$ Risk factors are heterogeneous and depend on the histological type of ovarian cancer. ${ }^{12}$ Various factors affect the occurrence of OC, of which genetic factors are among the most important. ${ }^{13}$

Currently, vulvar cancer (VUC) and vaginal cancer (VAC) are less prevalent, ranking, respectively, as the fifth (45,240 new cases, 17,427 deaths last year) and the sixth (17,908 new cases, 7995 deaths) most common cancers of the female reproductive system in terms of absolute numbers of cases worldwide. ${ }^{1}$ The worldwide distribution of vulvovaginal cancers is associated with less-developed regions as well as with advanced age, smoking, and HPV infection. ${ }^{14}$

The study aims to analyze and determine the incidence and mortality trends in gynecological cancers and BCs in Poland.

\section{Materials and Methods}

\section{Data Sources}

The study reviewed the entire female population in Poland experiencing gynecological cancers and BCs between 1980 and 2018. Incidence and mortality data for the investigated period were obtained from the Polish National Cancer 
Registry (PNCR). Cases of malignant neoplasms are collected in the PNCR according to malignant neoplasm reporting cards, and data on deaths caused by malignant neoplasms come from death certificates collected by the Central Statistical Office. ${ }^{15}$ Therefore, PNCR is a reliable source of data on cancer incidence and mortality in Poland. Between 1980 and 1996, diagnoses were classified according to the Ninth Revision of the International Classification of Diseases (ICD-9); after 1996, diagnoses were based on the Tenth Revision of the ICD (ICD-10). ${ }^{16}$ Cancers were coded as follows: BC as ICD-9 code 174 and ICD-10 code C50; CC as ICD-9 code 180 and ICD-10 code C53; CUC as ICD-9 code 182 and ICD10 code C54; OC as ICD-9 code 183 and ICD-10 code C56; VAC as ICD-10 code C52; and VUC as ICD-10 code C51. VAC and VUC did not have separate codes in the ICD-9 but instead were both coded as 184 . For this reason, they are included in our study only as of 1999. Regrettably, data for 1981 and 1986-1987 were unavailable for study, and data from 1997-1998 were missing because of medical staff strikes at that time. Institutional review board consent was not applicable, because anonymous registry data were retrospectively analyzed.

\section{Statistical Analysis}

The direct standardization method using the world standard population as reference one was used to calculate the agestandardized rates (ASRs) for cancer incidence and mortality. The direct standardization method was used because agespecific incidence and mortality rates were known. The denominator used in crude ratios and ASRs was 100,000. ${ }^{15}$ To determine trends, Joinpoint regression analysis was performed with the use of the Joinpoint Regression Program, version 4.8.0.1 (Statistical Methodology and Applications Branch, Surveillance Research Program, National Cancer Institute, Bethesda, MD). ${ }^{17}$ The analysis involved regression of the logarithmic time of the ASRs and then a transformation of the slope, resulting in the annual percentage change (APC). ${ }^{18}$ To consider various trends among different time partitions, the number of Joinpoints was set between 0 and 2, which allowed for the selection of a best-fitting model with the estimated APC for each segment. The average APC (AAPC) was used to illustrate the trend during the entire period under consideration. Trends were considered statistically significant when $\mathrm{p}$ values were $<0.05$. The term "stable" was used in the event that the APC was between -0.5 and 0.5. Below this interval, trends were deemed "decreasing"; above this interval, they were deemed "increasing." The above criteria for describing trends were applied in accordance with the guidelines of the National Cancer Institute. ${ }^{19}$ The trend data are presented as the APC or AAPC values with $95 \%$ confidence intervals (CIs). All calculations were performed using Joinpoint Regression Program, Version 4.8.0.1 (Statistical Research and Applications Branch, National Cancer Institute).

\section{Results \\ $\mathrm{BC}$}

Since 1980, 392,682 cases of BC have been diagnosed in Poland, and our analysis revealed a statistically significant increase in the incidence (AAPC: 2.3; CI: 1.8 to 2.9; $\mathrm{p}<0.05$ ). Overall, 163,202 Polish women have died from BC since 1980. However, despite the increased incidence, no statistically significant trend in mortality was noted during the study period (AAPC: 0.0; CI: -0.1 to 0.2; $\mathrm{p}=0.6$ ). Mortality increased during 1980-1994 and then decreased until 2010, at which time it began to increase again until the end of the observation period (Figure 1A, Table 1A).

Apart from women aged 20-24 years, each of the investigated 5-year age groups was characterized by a significant increase in the incidence of BC. The highest observed significant APC value was in women aged 70-74 years during 1988-1993 (APC: 7.63; CI: 3.4 to 12.1; $<<0.05$; Table 2A).

Mortality from BC has significantly decreased in recent years among women ages 25-34, 40-49, and 55-59 years, with APCs ranging from -2.46 to -1.26 . By contrast, mortality increased, as evidenced by statistically significant APCs occurring in older age groups (ie, those who were ages 50-54, 65-79, and $\geq 85$ years), with APC values ranging from 0.86 to 10.75 . Mortality from $\mathrm{BC}$ in the $60-64$ years age group did not show any significant trend during the entire timeframe under consideration. APC values for the 35-39 and 80-84 year age groups were also not significant (Table 1A). 


\section{CUC}

During 1980-2018, there were 128,112 cases of CUC, and 57,397 cases resulted in patient death. We observed an increase in CUC incidence (AAPC: 2.3 ; CI: 1.9 to 2.7 ; $\mathrm{p}<0.05$ ) during the study years, but mortality was stable (AAPC: 0.4; CI: 0.1 to $0.7 ; \mathrm{p}<0.05$ ); both trends were statistically significant. In the years 1980-2006, the CUC mortality rate had been decreasing. However, since 2006, mortality has been rapidly increasing, leading to an AAPC greater than zero (Figure 1A, Table 1A).

In nearly all 5-year age groups, CUC incidence increased during the study years. A nonsignificant decrease in the incidence of CUC was noted only in women aged 60-64 years since year 2008 (APC: $-0.61 ; \mathrm{Cl}:-1.6$ to $0.3 ; \mathrm{p}>0.05$ ). Almost all the increases in the incidence of CUC were statistically significant, except for a few time periods in the 55-59
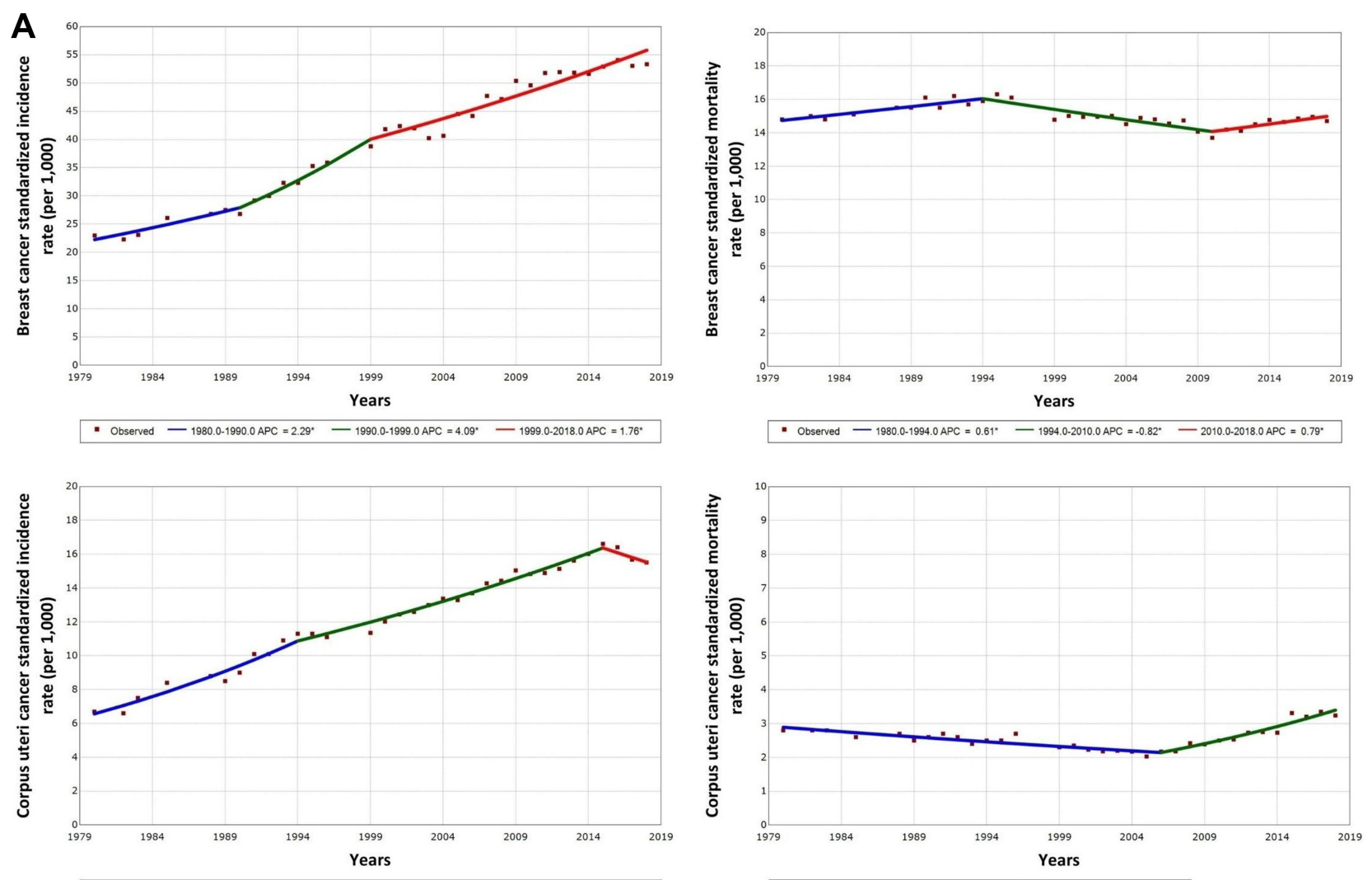

- Observed $-1980.0-1994.0 \mathrm{APC}=3.67^{\circ} \quad$ - $1994.0-2015.0 \mathrm{APC}=1.97^{\circ} \quad$-2015.0-2018.0 APC $=-1.74$

- Observed $-1980.0-2006.0 \mathrm{APC}=-1.14^{\circ} \quad-2006.0-2018.0 \mathrm{APC}=3.91^{\circ}$
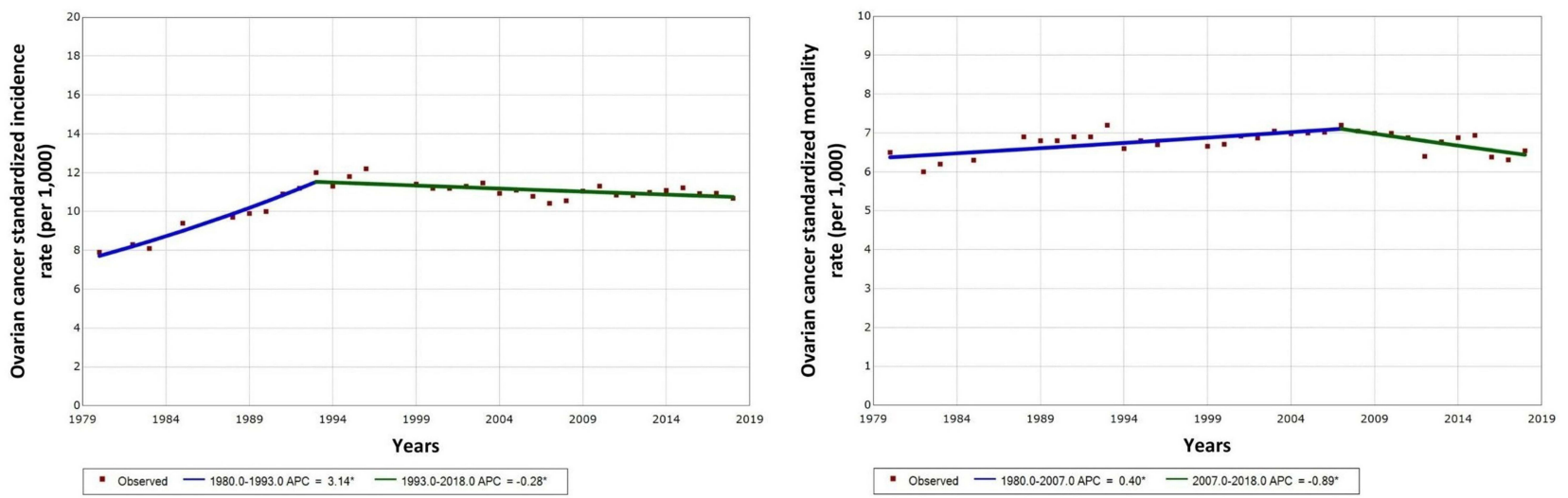

Figure I Continued. 

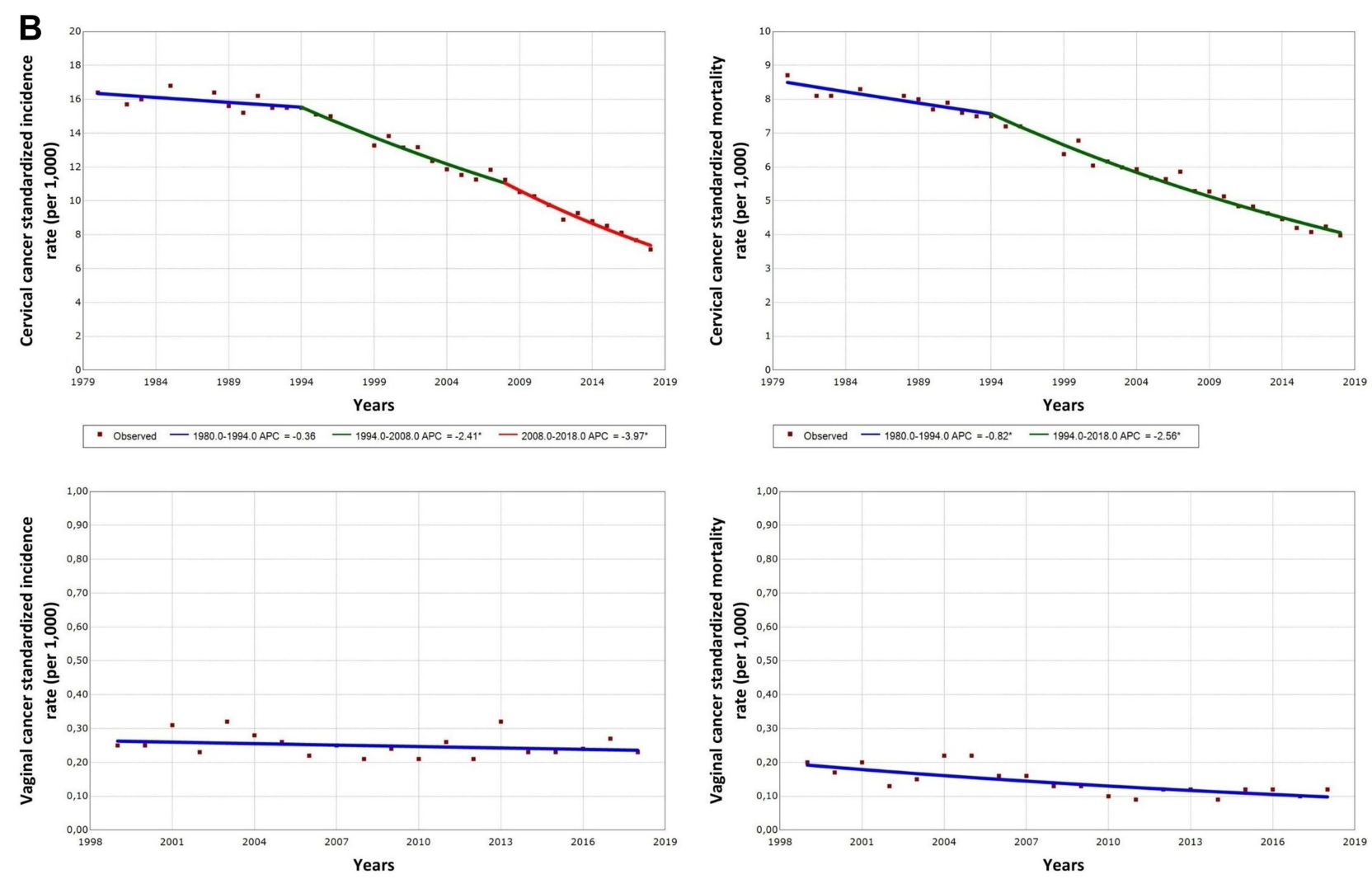

- Observed $-1999.0-2018.0 \mathrm{APC}=0.0 .57$
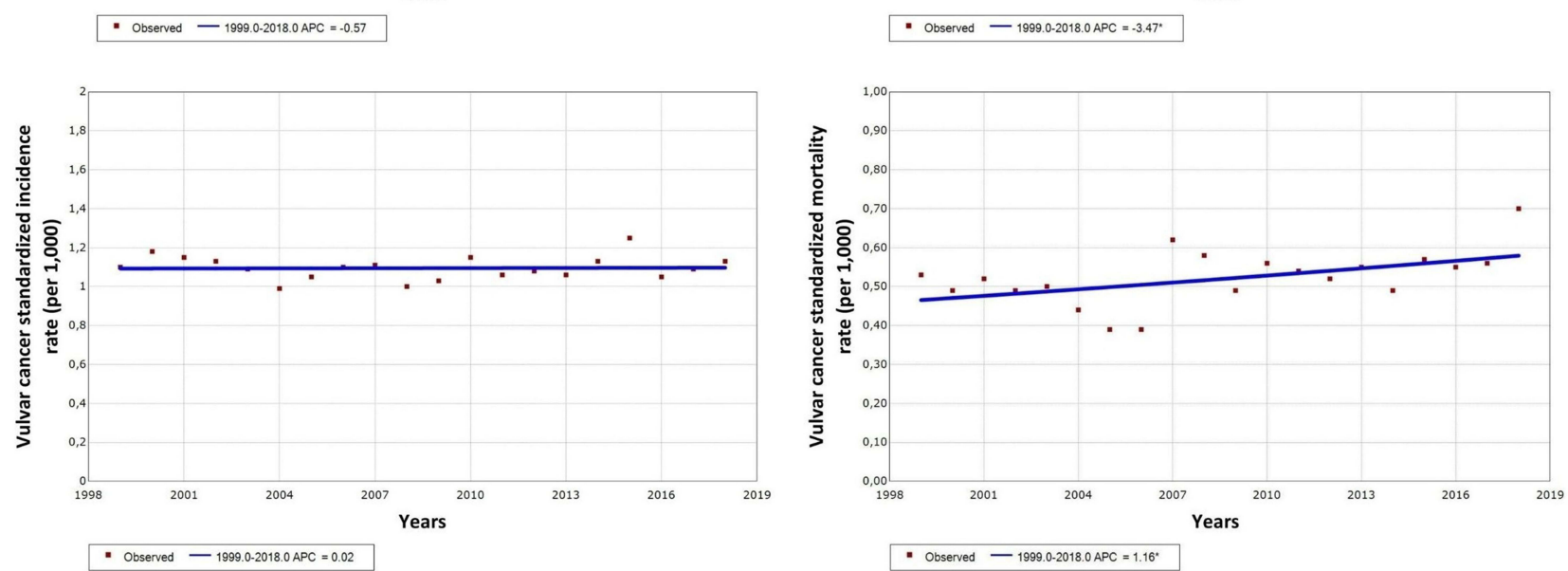

Figure I (A) Standardized incidence and mortality trends in breast, corpus uteri, and ovarian cancers, 1980-2018. (B) Standardized incidence and mortality trends in cervical, vaginal, and vulvar cancers, 1980-2018. *Statistically significant.

Abbreviations: APC, annual percentage change.

years and $\geq 85$ years age groups. The increase in the 55-59 years age group was no longer statistically significant after 2002 (APC: 0.46 ; $\mathrm{Cl}:-0.1$ to 1.0 ), and the increase in the $\geq 85$ years age group was not statistically significant during 1995-2004 (APC: 0.64; Cl: -3.4 to 4.9; Table 2A).

Mortality from CUC among women in their forties decreased significantly throughout the entire study period. Among women aged 50-79 years, mortality decreased in some 5-year age groups until the early 2000s. The declining trend was statistically significant for the 50-54 years age group (APC: $-2.70 ; \mathrm{Cl}:-3.5$ to -1.9 ), the $55-59$ years age group (APC: $-2.18 ; \mathrm{Cl}:-2.7$ to -1.7 ), and the $60-64$ years age group (APC $-1.09 ; \mathrm{Cl}:-1.8$ to -0.4 ). In contrast, from the beginning of the 2000s, a statistically significant increase in mortality was observed among women aged 50-79 years. The largest increase was detected in the 75-79 years age group (APC: 6.85 ; Cl: 4.7 to 9.1). Finally, during the entirety of the study 
period, mortality from CUC increased among women age $\geq 80$ years; this change was statistically significant as of the year 2007 both for the 80-84 years age group (APC: 8.40; Cl: 5.9 to 10.9) and the $\geq 85$ years age group (APC: 11.34; $\mathrm{Cl}$ : 7.5 to 15.3 ; Table $2 \mathrm{~A}$ ).

\section{Ovarian Cancer}

During the analyzed years, OC was diagnosed in 101,190 women, and 70,948 diagnosed cases ended in death. Between 1980 and 1993, OC incidence significantly increased and then stabilized, resulting in an overall AAPC greater than zero (AAPC: 0.9 ; CI: 0.7 to $1.1 ; \mathrm{p}<0.05$ ). Mortality was stable until 2007; then, it started to decrease and finally stabilized again, resulting in a non-statistically significant AAPC (AAPC: 0.0 ; CI: -0.2 to $0.2 ; \mathrm{p}=0.8$; Figure $1 \mathrm{~A}$, Table $1 \mathrm{~B}$ ).

A statistically significant decrease in the incidence of $\mathrm{OC}$ was observed in the youngest group (ages 10-14 years). A Joinpoint analysis identified an increase, followed by a decrease or stabilization, of OC incidence in women aged 40-59 years during the 1990s. However, women aged 65-79 years experienced a large initial increase and, thereafter, a lesser increase in OC incidence. The upward tendency became a nonsignificant, stable trend in the 60-65 years and $\geq 80$ years age groups. There were no significant trends in OC incidence among women aged 15-39 years (Table 2A).

Mortality from $\mathrm{OC}$ in the 5-year age groups showed a decrease among women age 20-54 years (statistically significant APC: -8.75 to -1.63 ), remaining stable for those age 60-64 years and increasing among older women. The statistically significant APCs for women $\geq 65$ years ranged from 0.91 to 3.30. There was no significant trend in OC mortality for the 55-59 years age group (Table $2 \mathrm{~A}$ ).

\section{Cervical Cancer}

From 1980 to 2018, 111,644 new cases of cervical cancer were diagnosed, and 57,397 women died. Both incidence and mortality decreased during those years (incidence AAPC: -2.1 ; CI: -2.3 to $-1.8 ; \mathrm{p}<0.05$; mortality AAPC: -2.0 ; CI: -2.2 to $-1.8 ; \mathrm{p}<0.05$; Figure 1B, Table 1B).

In the analysis of 5-year age groups, statistically significant, decreasing trends were observed in women aged 25-85 years during specific time frames. The decreasing trend was greatest among women ages 50-54 and 50-59 years (APC: -6.85 and -6.65 , respectively; $\mathrm{p}<0.05$ for both), whereas a statically significant, stable incidence trend was found only in the 50-54 years age group (APC: $-0.46 ; \mathrm{p}<0.05)$. The 40-44 years $(1980-1992)$ and $45-49$ years $(1980-2000)$ age groups experienced a significant trend of increasing incidence $(\mathrm{p}<0.05$; Table $2 \mathrm{~B})$.

Mortality from CC revealed a significant decrease among women aged 25-79 years in specific periods (APC: -6.95 and -0.58). There was no statistically significant, stable mortality trend. Only in 1980-1996, among women age 40-44 years, was there a statistically significant, increasing mortality trend (APC: $1.40 ; \mathrm{p}<0.05$; Table $2 \mathrm{~B}$ ).

\section{Vulvar Cancer}

During the 19-year study period, 9154 new cases of VUC were diagnosed, and 4698 deaths from VUC were confirmed. The incidence of VUC was stable during the analysis period (AAPC: 0.0 ; CI: -0.4 to $0.5 ; \mathrm{p}>0.05$ ). However, a significant increasing mortality trend was observed (AAPC: 1.16; CI: 0.1 to 2.2; $<<0.05$; Figure 1B, Table $1 \mathrm{C}$ ).

The analysis of the incidence of VUC showed decreasing trends in women aged 35-54 years, but only within the subgroups of ages 35-39 and 40-44 years was this trend statistically significant (APC: -5.30 and -5.01 , respectively; $\mathrm{p}<0.05$ for both). In the 55-59, 65-69, and 80-84 years age groups, incidence trends were stable ( $\mathrm{p}>0.05)$. Among women ages $60-64,70-74,75-79$, and $\geq 85$ years, increasing incidence trends were observed, although these changes were only statistically significant in the $70-74$ and $\geq 85$ years age groups (APC: 0.73 and 1.71, respectively; $<<0.05$ for both). The greatest increase was observed in the $\geq 85$ years age group, whereas the greatest decrease was observed in the 35-39 years age group $(\mathrm{p}<0.05$; Table $2 \mathrm{~B})$.

In 2015-2018, a major decrease in mortality was observed in women aged 50-54 years, but it was not statistically significant (APC: $-32.25 ; \mathrm{p}>0.05$ ). A slightly decreasing mortality trend was also observed in women aged $65-69$ years (APC: $-0.29 ; \mathrm{p}<0.05)$. The mortality trend was stable among women aged $65-79$ years $(\mathrm{p}>0.05)$. In 1999-2015, the mortality trend increased in the 50-54 years age group and increased during 1999-2018 in the 55-59, 60-64, 80-84, and 
$\geq 85$ age groups. The $\geq 85$ age group experienced the highest increase, and only this age group had statistically significant differences (APC: 2.64; $\mathrm{p}<0.05$; Table 2B).

\section{Vaginal Cancer}

Between 1999 and 2018, VAC was diagnosed in 1953 women, following a nonsignificant, decreasing incidence trend (AAPC: -0.6 ; CI: -1.6 to $0.5 ; \mathrm{p}>0.05$ ). During that time, 1247 women died of VAC, revealing a statistically significant, decreasing mortality trend (AAPC: -3.5 ; CI: -5.0 to -2.0 ; $<<0.05$; Figure 1B, Table $1 \mathrm{C}$ ).

The analysis of the incidence of VAC by 5 -year age groups showed decreasing incidence trends in women ages 50-54, 65-69 (1999-2014), 75-79, and 80-84 years; statistically significant decreasing incidence trends were detected in the 50-54, 65-69, and 80-84 years age groups (APC: $-3.91,-3.18$, and -2.70 , respectively; $\mathrm{p}<0.05$ ). Among women ages 55-59, 60-64, 65-69 (2014-2018), 70-74, and $\geq 85$ years, increasing incidence trends were observed (Table 2B).

In all groups, age-specific findings for mortality showed declining trends except the $\geq 85$ years age group. These declines were significant in the 50-54, 70-74, and 80-84 age groups (APC: $-4.85,-4.14$, and -3.41 , respectively; $\mathrm{p}<0.05$ for all). Among women in the $\geq 85$ years age group, the mortality trend was stable ( $>0.05$; Table $2 B$ ).

\section{Discussion}

$\mathrm{BC}$ consistently remains the most common malignancy of female genital organs in Poland, with an increasing incidence in nearly all age groups. Similar results have been reported about the incidence of CUC, although this trend has slowed in women aged 55-74 years.

According to the Main Statistical Office, the overall fertility rate in Poland has decreased from 2.2 in 1970 to 1.419 in 2019, whereas the mean age at first birth increased from age 22.8 years to 28.3 years in the same timeframe. ${ }^{20}$ Furthermore, the average age at menarche has decreased among cohorts of Polish women born between 1959-1970 and 1991-2001. ${ }^{21}$ Increasing age at first full-term pregnancy is a risk factor for BC, whereas a decreased birth rate, an earlier age at menarche, a history of late menopause, and use of menopausal hormonal therapy (depending on type of therapy) are risk factors for CUC, OC, and BC development. ${ }^{4,21-23}$ Furthermore, postponing pregnancy to an older reproductive age, which has become more common, may result in an increasing number of women diagnosed with the above-mentioned malignancies during pregnancy, as standard prenatal care allows for a concomitant incidental diagnosis of gynecological cancer or BC. ${ }^{24}$ All these factors combined may explain to some extent the incidence trends reported in our study. Changes in lifestyle, resulting in a higher prevalence of obesity, may also account for increasing trends in the incidence and mortality of CUC, BC, and OC. .,22,23,25 $^{2}$

Nationwide, a BC screening program was launched in 2006. According to assembled data, no significant changes have been observed since its launch. Nevertheless, the reported coverage of approximately $40 \%$ is low, which may also explain the persistently increasing mortality rates in older age groups, when diagnosis occurs at more advanced cancer stages. ${ }^{26}$ Declining mortality rates of BC, CUC, and OC have been observed generally among women younger than age 50 years. These observations may be related to regular gynecological care as a result of pregnancy or contraceptive use as well as to increasing awareness. Furthermore, new therapeutic options for BC, such as adjuvant radiotherapy, postsurgical hormonal treatment, and immunotherapy, have led to improvements in disease management. Nonetheless, some pharmaceutical agents have just been registered in Poland, so the definite effect of these novel options on trends in BC mortality remains to be determined. Recently HER2-targeted therapies have become accessible as part of the oncology drug program. Similarly, since 2021, olaparib has been refunded as first-line maintenance therapy in certain groups of women with the $B R C A 1 / 2$ mutation and $\mathrm{OC} .{ }^{27}$ Oral contraceptive use, which is a proven protective factor against epithelial OC - a type that comprises approximately $90 \%$ of all OC (the other $10 \%$ is represented by nonepithelial OC and amounts to a minor contributor to overall incidence and mortality)-likely has played a role in the incremental changes in OC incidence that, since the 1990s, has shown decreasing trends among women younger than age 60 years. ${ }^{23,28}$ This use also has resulted in either a slowing of the increase or stabilization of trends among older women. Similar changes in incidence trends have been reported in the United States. ${ }^{29}$ Generally, trends in OC incidence and mortality are comparable to those previously reported; however, a distinctively more decreasing trend in OC mortality has been recently observed in women aged $45-54$ years. Similarly, the continuation of formerly reported tendencies 
Table I Incidence and Mortality of Breast, Corpus Uteri (A), Ovarian, Cervical (B), and Vulvar and Vaginal (C) Cancers in Poland, $1980-2018$

\begin{tabular}{|c|c|c|c|c|c|c|c|c|c|c|c|c|}
\hline \multicolumn{13}{|c|}{ A. Breast, Corpus Uteri } \\
\hline \multirow[t]{3}{*}{ Years } & \multicolumn{6}{|c|}{ Breast Cancer } & \multicolumn{6}{|c|}{ Corpus Uteri Cancer } \\
\hline & \multicolumn{3}{|c|}{ Incidence } & \multicolumn{3}{|c|}{ Mortality } & \multicolumn{3}{|c|}{ Incidence } & \multicolumn{3}{|c|}{ Mortality } \\
\hline & $\mathbf{n}$ & CR & ASR & $\mathbf{n}$ & CR & ASR & $\mathbf{n}$ & CR & ASR & $\mathbf{n}$ & CR & ASR \\
\hline 1980 & 5154 & 28.3 & 23.0 & 3446 & 18.9 & 14.8 & 1524 & 8.4 & 6.7 & 702 & 3.9 & 2.8 \\
\hline 1981 & - & - & - & - & - & - & - & - & - & - & - & - \\
\hline 1982 & 5120 & 27.6 & 22.3 & 3641 & 19.6 & 15.0 & 1547 & 8.3 & 6.6 & 716 & 3.9 & 2.8 \\
\hline 1983 & 5386 & 28.7 & 23.1 & 3664 & 19.5 & 14.8 & 1796 & 9.6 & 7.5 & 726 & 3.9 & 2.8 \\
\hline 1984 & - & - & - & - & - & - & - & - & - & - & - & - \\
\hline 1985 & 6147 & 32.3 & 26.1 & 3795 & 19.9 & 15.1 & 2029 & 10.6 & 8.4 & 714 & 3.7 & 2.6 \\
\hline 1986 & - & - & - & - & - & - & - & - & - & - & - & - \\
\hline 1987 & - & - & - & - & - & - & - & - & - & - & - & - \\
\hline 1988 & 6513 & 33.6 & 26.8 & 4045 & 20.9 & 15.5 & 2200 & 11.3 & 8.8 & 758 & 2.9 & 2.7 \\
\hline 1989 & 6706 & 34.5 & 27.5 & 4097 & 21.1 & 15.5 & 2166 & 11.1 & 8.5 & 702 & 3.6 & 2.5 \\
\hline 1990 & 6649 & 34.0 & 26.8 & 4323 & 22.1 & 16.1 & 2301 & 11.8 & 9.0 & 763 & 3.9 & 2.6 \\
\hline 1991 & 7343 & 37.4 & 29.2 & 4198 & 21.4 & 15.5 & 2626 & 13.4 & 10.1 & 776 & 4.0 & 2.7 \\
\hline 1992 & 7671 & 29.0 & 30.0 & 4429 & 22.5 & 16.2 & 2611 & 13.3 & 10.1 & 781 & 4.0 & 2.6 \\
\hline 1993 & 8416 & 42.6 & 32.3 & 4381 & 22.2 & 15.7 & 2858 & 14.5 & 10.9 & 724 & 3.7 & 2.4 \\
\hline 1994 & 8458 & 42.8 & 32.3 & 4449 & 22.5 & 15.9 & 3001 & 15.2 & 11.3 & 762 & 3.9 & 2.5 \\
\hline 1995 & 9173 & 46.3 & 35.3 & 4665 & 23.6 & 16.3 & 2980 & 15.0 & 11.3 & 763 & 3.9 & 2.5 \\
\hline 1996 & 9681 & 48.8 & 35.9 & 4738 & 23.9 & 16.1 & 3043 & 15.3 & II.I & 845 & 4.2 & 2.7 \\
\hline 1997 & - & - & - & - & - & - & - & - & - & - & - & - \\
\hline 1998 & - & - & - & - & - & - & - & - & - & - & - & - \\
\hline 1999 & 10,903 & 54.9 & 38.8 & 4553 & 22.9 & 14.8 & 3260 & 16.4 & 11.4 & 761 & 3.8 & 2.3 \\
\hline 2000 & 11,853 & 59.7 & 41.8 & 4712 & 23.7 & 15.0 & 3496 & 17.6 & 12.0 & 808 & 4.1 & 2.4 \\
\hline 2001 & 12,118 & 61.0 & 42.4 & 4825 & 24.3 & 15.0 & 3675 & 18.5 & 12.4 & 776 & 3.9 & 2.2 \\
\hline 2002 & 12,132 & 61.5 & 42.0 & 4825 & 24.5 & 15.0 & 3796 & 19.3 & 12.6 & 757 & 3.8 & 2.2 \\
\hline 2003 & 11,733 & 59.6 & 40.2 & 4942 & 25.1 & 15.0 & 3953 & 20.1 & 13.0 & 783 & 4.0 & 2.2 \\
\hline 2004 & 12,049 & 61.2 & 40.7 & 4887 & 24.8 & 14.5 & 4193 & 21.3 & 13.4 & 794 & 4.0 & 2.2 \\
\hline 2005 & 13,385 & 67.9 & 44.5 & 5112 & 26.0 & 14.9 & 4196 & 21.3 & 13.3 & 770 & 3.9 & 2.0 \\
\hline 2006 & 13,322 & 67.6 & 44.2 & 5212 & 26.5 & 14.8 & 4376 & 22.2 & 13.7 & 814 & 4.1 & 2.2 \\
\hline 2007 & 14,484 & 73.5 & 47.7 & 5255 & 26.7 & 14.6 & 4640 & 23.6 & 14.3 & 848 & 4.3 & 2.2 \\
\hline 2008 & 14,576 & 74.0 & 47.2 & 5362 & 27.2 & 14.7 & 4820 & 24.5 & 14.4 & 952 & 4.8 & 2.4 \\
\hline
\end{tabular}

(Continued) 
Table I (Continued).

\begin{tabular}{|c|c|c|c|c|c|c|c|c|c|c|c|c|}
\hline \multicolumn{13}{|c|}{ A. Breast, Corpus Uteri } \\
\hline \multirow[t]{3}{*}{ Years } & \multicolumn{6}{|c|}{ Breast Cancer } & \multicolumn{6}{|c|}{ Corpus Uteri Cancer } \\
\hline & \multicolumn{3}{|c|}{ Incidence } & \multicolumn{3}{|c|}{ Mortality } & \multicolumn{3}{|c|}{ Incidence } & \multicolumn{3}{|c|}{ Mortality } \\
\hline & $\mathbf{n}$ & CR & ASR & $\mathbf{n}$ & CR & ASR & $\mathbf{n}$ & CR & ASR & $\mathbf{n}$ & CR & ASR \\
\hline 2009 & 15,752 & 79.8 & 50.4 & 5241 & 26.6 & 14.1 & 5061 & 25.7 & 15.0 & 969 & 4.9 & 2.4 \\
\hline 2010 & 15,784 & 79.4 & 49.6 & 5226 & 26.3 & 13.7 & 5125 & 25.8 & 14.8 & 1042 & 5.2 & 2.5 \\
\hline 2011 & 16,534 & 83.2 & 51.8 & 5437 & 27.4 & 14.2 & 5251 & 26.4 & 14.9 & 1085 & 5.5 & 2.5 \\
\hline 2012 & 17,000 & 85.7 & 51.9 & 5574 & 28.1 & 14.1 & 5426 & 27.4 & 15.1 & 1162 & 5.9 & 2.7 \\
\hline 2013 & 17,142 & 86.3 & 51.8 & 5816 & 29.3 & 14.5 & 5706 & 28.7 & 15.6 & 1243 & 6.3 & 2.8 \\
\hline 2014 & 17,379 & 87.5 & 51.6 & 5975 & 30.1 & 14.8 & 5944 & 29.9 & 16.0 & 1280 & 6.4 & 2.7 \\
\hline 2015 & 18,106 & 91.2 & 52.9 & 6319 & 31.8 & 14.6 & 6243 & 31.5 & 16.6 & 1690 & 8.5 & 3.3 \\
\hline 2016 & 18,615 & 93.9 & 54.1 & 6493 & 32.7 & 14.9 & 6226 & 31.6 & 16.4 & 1600 & 8.1 & 3.2 \\
\hline 2017 & 18,529 & 93.4 & 53.0 & 6670 & 33.6 & 15.0 & 5984 & 30.2 & 15.7 & 1761 & 8.9 & 3.4 \\
\hline 2018 & 18,869 & 95.2 & 53.3 & 6895 & 34.8 & 14.7 & 6059 & 30.6 & 15.6 & $|78|$ & 9.0 & 3.2 \\
\hline \multicolumn{13}{|c|}{ B. Ovarian, Cervical } \\
\hline \multirow[t]{3}{*}{ Years } & \multicolumn{6}{|c|}{ Ovarian Cancer } & \multicolumn{6}{|c|}{ Cervical Cancer } \\
\hline & \multicolumn{3}{|c|}{ Incidence } & \multicolumn{3}{|c|}{ Mortality } & \multicolumn{3}{|c|}{ Incidence } & \multicolumn{3}{|c|}{ Mortality } \\
\hline & $\mathbf{n}$ & CR & ASR & $\mathbf{n}$ & CR & ASR & $n$ & CR & ASR & $n$ & CR & ASR \\
\hline 1980 & 1734 & 9.5 & 7.9 & 1464 & 8.0 & 6.5 & 3539 & 19.4 & 16.4 & 1997 & 11.0 & 8.7 \\
\hline I98I & - & - & - & - & - & - & - & - & - & - & - & - \\
\hline 1982 & 1830 & 9.9 & 8.3 & 1397 & 7.5 & 6.0 & 3473 & 18.7 & 15.7 & 1913 & 10.3 & 8.1 \\
\hline 1983 & 1856 & 9.9 & 8.1 & 1478 & 7.9 & 6.2 & 3612 & 19.3 & 16.0 & 1946 & 10.4 & 8.1 \\
\hline 1984 & - & - & - & - & - & - & - & - & - & - & - & - \\
\hline 1985 & 2150 & 11.3 & 9.4 & 1522 & 8.0 & 6.3 & 3843 & 20.2 & 16.8 & 2028 & 10.6 & 8.3 \\
\hline 1986 & - & - & - & - & - & - & - & - & - & - & - & - \\
\hline 1987 & - & - & - & - & - & - & - & - & - & - & - & - \\
\hline 1988 & 2333 & 12.0 & 9.7 & $|73|$ & 8.9 & 6.9 & 3893 & 20.1 & 16.4 & 2063 & 10.6 & 8.1 \\
\hline 1989 & 2370 & 12.2 & 9.9 & 1718 & 8.8 & 6.8 & 3764 & 19.4 & 15.6 & 2020 & 10.4 & 8.0 \\
\hline 1990 & 2404 & 12.3 & 10.0 & 1747 & 8.9 & 6.8 & 3658 & 18.7 & 15.2 & 1981 & 10.1 & 7.7 \\
\hline 1991 & 2680 & 13.7 & 10.9 & 1787 & 9.1 & 6.9 & 3954 & 20.2 & 16.2 & 2070 & 10.6 & 7.9 \\
\hline 1992 & 2765 & 14.1 & 11.2 & 1850 & 9.2 & 6.9 & 3832 & 19.5 & 15.5 & 1997 & 10.1 & 7.6 \\
\hline 1993 & 2982 & 15.1 & 12.0 & 1880 & 9.5 & 7.2 & 3903 & 19.8 & 15.5 & 2028 & 10.3 & 7.5 \\
\hline 1994 & 2876 & 14.5 & 11.3 & 1794 & 9.1 & 6.6 & 3943 & 19.9 & 15.5 & 2032 & 10.3 & 7.5 \\
\hline 1995 & 2993 & 15.1 & 11.8 & 1840 & 9.3 & 6.8 & 3856 & 19.5 & $15 . \mid$ & 1992 & 10.1 & 7.2 \\
\hline
\end{tabular}

(Continued) 
Table I (Continued).

\begin{tabular}{|c|c|c|c|c|c|c|c|c|c|c|c|c|}
\hline \multicolumn{13}{|c|}{ B. Ovarian, Cervical } \\
\hline \multirow[t]{3}{*}{ Years } & \multicolumn{6}{|c|}{ Ovarian Cancer } & \multicolumn{6}{|c|}{ Cervical Cancer } \\
\hline & \multicolumn{3}{|c|}{ Incidence } & \multicolumn{3}{|c|}{ Mortality } & \multicolumn{3}{|c|}{ Incidence } & \multicolumn{3}{|c|}{ Mortality } \\
\hline & $\mathbf{n}$ & CR & ASR & $\mathbf{n}$ & CR & ASR & $\mathbf{n}$ & CR & ASR & $\mathbf{n}$ & CR & ASR \\
\hline 1996 & 3220 & 16.2 & 12.2 & 1909 & 9.6 & 6.7 & 3990 & 19.6 & 15.0 & 2025 & 10.2 & 7.2 \\
\hline 1997 & - & - & - & - & - & - & - & - & - & - & - & - \\
\hline 1998 & - & - & - & - & - & - & - & - & - & - & - & - \\
\hline 1999 & 3151 & 15.9 & 11.4 & 1959 & 9.9 & 6.7 & 3565 & 18.0 & 13.3 & 1859 & 9.4 & 6.4 \\
\hline 2000 & 3157 & 15.9 & 11.2 & 2032 & 10.2 & 6.7 & 3777 & 19.0 & 13.8 & 1987 & 10.0 & 6.8 \\
\hline 2001 & 3193 & 16.1 & 11.2 & 2152 & 10.8 & 6.9 & 3604 & 18.1 & 13.2 & 1826 & 9.2 & 6.0 \\
\hline 2002 & 3267 & 16.6 & 11.3 & 2171 & 11.0 & 6.9 & 3610 & 18.3 & 13.2 & 1855 & 9.4 & 6.2 \\
\hline 2003 & 3371 & 17.1 & 11.5 & 2271 & 11.5 & 7.1 & 3439 & 17.5 & 12.4 & 1825 & 9.3 & 6.0 \\
\hline 2004 & 3264 & 16.6 & 10.9 & 2273 & 11.5 & 7.0 & 3345 & 17.0 & 11.9 & 1819 & 9.2 & 5.9 \\
\hline 2005 & 3355 & 17.0 & II.I & 2357 & 12.0 & 7.0 & 3263 & 16.6 & 11.5 & 1796 & 9.1 & 5.7 \\
\hline 2006 & 3291 & 16.7 & 10.8 & 2390 & 12.1 & 7.0 & 3226 & 16.4 & 11.3 & 1824 & 9.3 & 5.6 \\
\hline 2007 & 3214 & 16.3 & 10.4 & 2485 & 12.6 & 7.2 & 3431 & 17.4 & 11.8 & 1907 & 9.7 & 5.9 \\
\hline 2008 & 3280 & 16.6 & 10.6 & 2507 & 12.7 & 7.1 & 3270 & 16.6 & 11.2 & 1745 & 8.9 & 5.3 \\
\hline 2009 & 3474 & 17.6 & II.I & 2510 & 12.7 & 7.0 & 3102 & 15.7 & 10.5 & 1748 & 8.9 & 5.3 \\
\hline 2010 & 3587 & 18.1 & 11.3 & 2547 & 12.8 & 7.0 & 3078 & 15.5 & 10.3 & 1735 & 8.7 & 5.1 \\
\hline 2011 & 3527 & 17.8 & 10.9 & 2558 & 12.9 & 6.9 & 2968 & 14.9 & 9.8 & 1656 & 8.3 & 4.8 \\
\hline 2012 & 3544 & 17.9 & 10.8 & 2432 & 12.3 & 6.4 & 2783 & 14.0 & 8.9 & 1669 & 8.4 & 4.8 \\
\hline 2013 & 3639 & 18.3 & 11.0 & 2603 & 13.1 & 6.8 & 2909 & 14.6 & 9.3 & 1669 & 8.4 & 4.6 \\
\hline 2014 & 3722 & 18.7 & II.I & 2678 & 13.5 & 6.9 & 2807 & 14.1 & 8.8 & 1628 & 8.2 & 4.5 \\
\hline 2015 & 3735 & 18.8 & 11.2 & 2768 & 14.0 & 6.9 & 2723 & 13.7 & 8.5 & 1585 & 8.0 & 4.2 \\
\hline 2016 & 3717 & 18.7 & 10.9 & 2639 & 13.3 & 6.4 & 2622 & 13.2 & 8.1 & 1570 & 7.9 & 4.1 \\
\hline 2017 & 3775 & 19.0 & 10.9 & 2670 & 13.5 & 6.3 & 2502 & 12.6 & 7.7 & 1609 & 8.1 & 4.2 \\
\hline 2018 & 3734 & 18.8 & 10.7 & 2829 & 14.3 & 6.5 & 2360 & 11.9 & 7.1 & 1593 & 8.0 & 4.0 \\
\hline \multicolumn{13}{|c|}{ C. Vulvar and Vaginal } \\
\hline \multirow[t]{3}{*}{ Years } & \multicolumn{6}{|c|}{ Vulvar Cancer } & \multicolumn{6}{|c|}{ Vaginal Cancer } \\
\hline & \multicolumn{3}{|c|}{ Incidence } & \multicolumn{3}{|c|}{ Mortality } & \multicolumn{3}{|c|}{ Incidence } & \multicolumn{3}{|c|}{ Mortality } \\
\hline & $\mathbf{n}$ & CR & ASR & $\mathbf{n}$ & CR & ASR & $\mathbf{N}$ & CR & ASR & $\mathbf{n}$ & CR & ASR \\
\hline 1999 & 378 & 1.90 & 1.10 & 212 & 1.07 & 0.53 & 85 & 0.43 & 0.25 & 67 & 0.34 & 0.20 \\
\hline 2000 & 406 & 2.04 & 1.18 & 195 & 0.98 & 0.49 & 89 & 0.45 & 0.25 & 66 & 0.33 & 0.17 \\
\hline 2001 & 420 & 2.11 & 1.15 & 207 & 1.04 & 0.52 & 102 & 0.51 & 0.31 & 73 & 0.37 & 0.20 \\
\hline
\end{tabular}

(Continued) 
Table I (Continued).

\begin{tabular}{|c|c|c|c|c|c|c|c|c|c|c|c|c|}
\hline \multicolumn{13}{|c|}{ C. Vulvar and Vaginal } \\
\hline \multirow[t]{3}{*}{ Years } & \multicolumn{6}{|c|}{ Vulvar Cancer } & \multicolumn{6}{|c|}{ Vaginal Cancer } \\
\hline & \multicolumn{3}{|c|}{ Incidence } & \multicolumn{3}{|c|}{ Mortality } & \multicolumn{3}{|c|}{ Incidence } & \multicolumn{3}{|c|}{ Mortality } \\
\hline & $\mathbf{n}$ & CR & ASR & $\mathbf{n}$ & CR & ASR & $\mathbf{N}$ & CR & ASR & $\mathbf{n}$ & CR & ASR \\
\hline 2002 & 407 & 2.06 & 1.13 & 203 & 1.03 & 0.49 & 84 & 0.43 & 0.23 & 48 & 0.24 & 0.13 \\
\hline 2003 & 408 & 2.07 & 1.09 & 213 & 1.08 & 0.50 & 111 & 0.56 & 0.32 & 61 & 0.31 & 0.15 \\
\hline 2004 & 382 & 1.94 & 0.99 & 194 & 0.98 & 0.44 & 103 & 0.52 & 0.28 & 80 & 0.41 & 0.22 \\
\hline 2005 & 406 & 2.06 & 1.05 & 169 & 0.86 & 0.39 & 95 & 0.48 & 0.26 & 92 & 0.47 & 0.22 \\
\hline 2006 & 419 & 2.14 & 1.10 & 186 & 0.94 & 0.39 & 87 & 0.44 & 0.22 & 67 & 0.34 & 0.16 \\
\hline 2007 & 439 & 2.23 & 1.11 & 280 & 1.42 & 0.62 & 95 & 0.48 & 0.25 & 81 & 0.41 & 0.16 \\
\hline 2008 & 414 & 2.10 & 1.00 & 265 & 1.34 & 0.58 & 84 & 0.43 & 0.21 & 55 & 0.28 & 0.13 \\
\hline 2009 & 436 & 2.21 & 1.03 & 232 & 1.18 & 0.49 & 85 & 0.43 & 0.24 & 53 & 0.27 & 0.13 \\
\hline 2010 & 491 & 2.47 & 1.15 & 273 & 1.37 & 0.56 & 83 & 0.42 & 0.21 & 45 & 0.23 & 0.10 \\
\hline 2011 & 463 & 2.33 & 1.06 & 270 & 1.36 & 0.54 & 101 & 0.51 & 0.26 & 44 & 0.22 & 0.09 \\
\hline 2012 & 489 & 2.46 & 1.08 & 273 & 1.37 & 0.52 & 84 & 0.42 & 0.21 & 57 & 0.29 & 0.12 \\
\hline 2013 & 490 & 2.47 & 1.06 & 303 & 1.53 & 0.55 & 136 & 0.68 & 0.32 & 63 & 0.32 & 0.12 \\
\hline 2014 & 515 & 2.59 & 1.13 & 267 & 1.34 & 0.49 & 107 & 0.54 & 0.23 & 42 & 0.21 & 0.09 \\
\hline 2015 & 581 & 2.93 & 1.25 & 315 & 1.59 & 0.57 & 104 & 0.52 & 0.23 & 65 & 0.33 & 0.12 \\
\hline 2016 & 498 & 2.51 & 1.05 & 326 & 1.64 & 0.55 & 106 & 0.53 & 0.24 & 64 & 0.32 & 0.12 \\
\hline 2017 & 539 & 2.72 & 1.09 & 315 & 1.59 & 0.56 & 106 & 0.53 & 0.27 & 56 & 0.28 & 0.10 \\
\hline 2018 & 573 & 2.89 & 1.13 & 3.19 & 1.61 & 0.70 & 106 & 0.53 & 0.23 & 68 & 0.34 & 0.12 \\
\hline
\end{tabular}

Notes: Missing data for 1981, 1985-1986, and 1997-1998. No data on vulvar and vaginal cancers before 1999 are available because of the lack of separate codes for them in the International Classification of Diseases, 9th revision.

Abbreviation: ASR - age-standardized ratio; CR - crude ratio.

regarding $\mathrm{BC}$ incidence has been observed. Nonetheless, increasing trends have become more pronounced in women ages 30-34, 70-74, and 80-84 years, whereas stable trends have appeared in ages 55-59 and 65-69 years. BC Increasing mortality trends have been observed in women older than age 65 years and in women of all ages since approximately the beginning of the second decade of the 21 st century. ${ }^{30}$ Nevertheless, $\mathrm{BC}$ incidence and mortality trends in Poland are worse compared with those of other countries in the European Union or elsewhere in the world. ${ }^{31-33}$ Unfavorable results of comparisons between Poland and other European countries regarding trends in BC mortality have also been presented in a study by Wojtyla et al that covered 1980-2017. The results of BC mortality trend analysis of that study in all ages are in line with findings presented in our study; both studies showed stable trends during the entire study period and an increasing trend starting from approximately 2010. However, Wojtyla et al did not analyze trends in BC mortality by 5year age groups. ${ }^{34}$ According to Carioli et al, reduction in overall $\mathrm{BC}$ mortality was the smallest within the European Union; similarly, in a study by Malvezzi, OC mortality in 2002-2012 showed a small reduction. ${ }^{35,36}$ In the first decade of the 21st century, a reversal in the CUC mortality trend, from declining or stable to significantly increasing or an acceleration of an increasing trend, was found in all women age $\geq 50$ years. Similar tendencies have been observed in other highly developed countries, and the trends generally correlate with the human development index. ${ }^{37}$ In the United 
Table 2 Age-Specific Incidence and Mortality of Breast, Corpus Uteri, Ovarian (A), Cervical, Vulvar, and Vaginal (B) Cancers in Poland, I980-2018

\begin{tabular}{|c|c|c|c|c|c|c|}
\hline \multicolumn{7}{|c|}{ A. Breast, Corpus Uteri, Ovarian } \\
\hline \multirow[t]{2}{*}{ Age, years } & \multicolumn{2}{|c|}{ Breast Cancer Trends } & \multicolumn{2}{|c|}{ Corpus Uteri Cancer Trends } & \multicolumn{2}{|c|}{ Ovarian Cancer Trends } \\
\hline & $\begin{array}{c}\text { Incidence, APC } \\
(95 \% \mathrm{Cl})\end{array}$ & $\begin{array}{c}\text { Mortality, APC, } \\
(95 \% \mathrm{Cl})\end{array}$ & $\begin{array}{c}\text { Incidence, APC } \\
(95 \% \mathrm{Cl})\end{array}$ & $\begin{array}{c}\text { Mortality, APC } \\
(95 \% \mathrm{Cl})\end{array}$ & $\begin{array}{c}\text { Incidence, APC } \\
(95 \% \mathrm{Cl})\end{array}$ & $\begin{array}{c}\text { Mortality, APC } \\
(95 \% \mathrm{Cl})\end{array}$ \\
\hline $0-4$ & - & - & - & - & - & - \\
\hline $5-9$ & - & - & - & - & - & - \\
\hline $10-14$ & - & - & - & - & $\begin{array}{l}\text { |980-20|8: }-\left.2.0\right|^{*} \\
\quad(-3.5 \text { to }-0.5)\end{array}$ & - \\
\hline $15-19$ & - & - & - & - & $\begin{array}{l}\text { 1980-2018: }-0.44 \\
(-1.7 \text { to } 0.8)\end{array}$ & - \\
\hline $20-24$ & 1980-2018: $-1.38 *(-2.6$ to -0.1$)$ & - & - & - & $\begin{array}{l}\text { |980-2018: }-0.33 \\
\quad(-1.1 \text { to } 0.4)\end{array}$ & $\begin{array}{l}\text { 1980-2018: }-3.15^{*} \\
\quad(-5.1 \text { to }-1.2)\end{array}$ \\
\hline $25-29$ & 1980-2018: $1.37^{*}$ (0.8 to 2.0$)$ & $\begin{array}{c}\text { 1980-2018: }-2.43 * \\
(-3.8 \text { to }-1.0)\end{array}$ & - & - & $\begin{array}{l}\text { 1980-20II: }-0.32 \\
(-1.2 \text { to } 0.5) \\
2011-2018: 6.32 \\
(-1.0 \text { to } 14.2)\end{array}$ & $\begin{array}{l}\text { 1980-20|8: }-3.50^{*} \\
\quad(-4.8 \text { to }-2.2)\end{array}$ \\
\hline $30-34$ & $\begin{array}{l}\text { I980-1996: }-0.09(-1.3 \text { to } 1.2) \\
\text { I996-20I8: } 2.67 *(1.9 \text { to } 3.5)\end{array}$ & $\begin{array}{l}\text { 1980-2018: }-1.26 * \\
(-1.9 \text { to }-0.6)\end{array}$ & $\begin{array}{l}\text { |980-20|8: } 2.29 * \\
\quad(0.7 \text { to } 3.8)\end{array}$ & - & $\begin{array}{l}\text { |980-2018: }-0.43 \\
(-0.9 \text { to } 0.0)\end{array}$ & $\begin{array}{c}\text { 1980-1988: } 6.44 \\
(-7.3 \text { to } 22.3) \\
\text { 1988-20|8: }-3.82 * \\
(-4.8 \text { to }-2.8)\end{array}$ \\
\hline $35-39$ & 1980-20|8: I.70* (I.5 to I.9) & $\begin{array}{c}\text { 1980-2010: }-2.46 * \\
(-3.1 \text { to }-1.8) \\
2010-2018: 1.63 \\
(-2.5 \text { to } 5.9)\end{array}$ & $\begin{array}{l}\text { |980-20|8: }\left.1.7\right|^{*} \\
\quad(1.0 \text { to } 2.4)\end{array}$ & - & $\begin{array}{l}\text { 1980-2018: }-0.26 \\
(-0.7 \text { to } 0.1)\end{array}$ & $\begin{array}{l}\text { 1980-20|8: }-2.72 * \\
(-3.2 \text { to }-2.3)\end{array}$ \\
\hline $40-44$ & $\begin{array}{l}\text { I980-1988: } 4.66 *(2.2 \text { to } 7.2) \\
\text { I988-20|8: } 1.34 *(1.2 \text { to } 1.5)\end{array}$ & $\begin{array}{c}\text { 1980-1990: } 1.44 \\
(-1.2 \text { to } 4.1) \\
1990-2018:-2.20 * \\
(-2.7 \text { to }-1.7)\end{array}$ & $\begin{array}{l}\text { 1980-2018: } 1.47^{*} \\
\quad(1.1 \text { to } 1.9)\end{array}$ & $\begin{array}{c}\text { |980-20|8: }-3.21 *(-4.1 \\
\text { to }-2.3)\end{array}$ & $\begin{array}{c}|980-199|: 3.54^{*} \\
(1.9 \text { to } 5.2) \\
|99|-2018:-1.31 * \\
(-1.7 \text { to }-0.9)\end{array}$ & $\begin{array}{c}\text { 1980-1993: } 1.44 \\
(-0.6 \text { to } 3.5) \\
\text { 1993-2018: }-3.01 * \\
(-3.7 \text { to }-2.3)\end{array}$ \\
\hline
\end{tabular}




\begin{tabular}{|c|c|c|c|c|c|c|}
\hline $45-49$ & $\begin{array}{l}\text { 1980-1996: } 4.06 *(3.3 \text { to } 4.8) \\
\text { 1996-2018: } 0.76 * \text { (0.3 to I.2) }\end{array}$ & $\begin{array}{l}\text { 1980-1994: } 0.57 \\
(-0.4 \text { to } 1.6) \\
\text { 1994-20|8: }-2.16^{*} \\
(-2.6 \text { to }-1.7)\end{array}$ & $\begin{array}{l}\text { 1980-2018: } 1.35^{*} \\
\quad(1.0 \text { to } 1.7)\end{array}$ & $\begin{array}{c}\text { 1980-2018: }-3.04 *(-3.9 \\
\text { to }-2.1)\end{array}$ & $\begin{array}{l}\text { 1980-1993: } 3.22^{*} \\
(2.3 \text { to } 4.1) \\
\text { 1993-2018: }-0.96^{*} \\
(-1.3 \text { to }-0.7)\end{array}$ & $\begin{array}{c}\text { 1980-1992: } 0.81 \\
(-1.0 \text { to } 2.6) \\
\text { 1992-2014: }-1.63^{*} \\
(-2.3 \text { to }-0.9) \\
2014-1018:-8.75^{*} \\
(-16.2 \text { to }-0.7)\end{array}$ \\
\hline 50-54 & $\begin{array}{l}\text { 1980-1990: } 1.30 \text { (0.0 to } 2.7) \\
\text { 1990-2000: } 6.42^{*}(4.7 \text { to } 8.1) \\
\text { 2000-2018: } 0.63^{*}(0.2 \text { to } 1.1)\end{array}$ & $\begin{array}{l}\text { 1980-1995: } 0.37 \\
(-0.4 \text { to } 1.2) \\
\text { 1995-2018: } 1.63^{*} \\
(-2.0 \text { to }-1.2)\end{array}$ & $\begin{array}{l}\text { 1980-1993: }\left.3.5\right|^{*} \\
(2.5 \text { to } 4.6) \\
\text { 1993-2018: } 0.73^{*} \\
(0.4 \text { to } 1.1)\end{array}$ & $\begin{array}{c}\text { 1980-2008: }-2.70 *(-3.5 \\
\text { to }-1.9) \\
2008-2018: 2.44(-1.0 \text { to } \\
6.0)\end{array}$ & $\begin{array}{l}\text { 1980-1995: } 3.16^{*} \\
(2.3 \text { to } 4.0) \\
1995-2018:-1.02 * \\
(-1.5 \text { to }-0.6)\end{array}$ & $\begin{array}{c}\text { 1980-2006: } 0.21 \\
(-0.3 \text { to } 0.8) \\
2006-2018:-\left.2.2\right|^{*} \\
(-3.7 \text { to }-0.7)\end{array}$ \\
\hline 55-59 & $\begin{array}{l}\text { 1980-1992: 1.58* (0.5 to } 2.6) \\
\text { 1992-2002: } 6.54^{*}(5.0 \text { to } 8.1) \\
\text { 2002-2018: } 0.09 \text { (-0.5 to } 0.7)\end{array}$ & $\begin{array}{l}\text { 1980-2003: } 0.18 \\
(-0.2 \text { to } 0.5) \\
2003-2018:-1.43^{*} \\
(-1.9 \text { to }-0.8)\end{array}$ & $\begin{array}{l}\text { 1980-2002: } 2.43^{*} \\
\quad(2.0 \text { to } 2.9) \\
2002-2018: 0.46 \\
(-0.1 \text { to } 1.0)\end{array}$ & $\begin{array}{c}\text { 1980-2006: }-2.18^{*}(-2.7 \\
\text { to }-1.7) \\
\text { 2006-20।8: } 2.80^{*} \text { (1.4 to } \\
4.3)\end{array}$ & $\begin{array}{l}\text { 1980-1994: } 3.30^{*} \\
(2.6 \text { to } 4.0) \\
\text { 1994-2018: }-0.38^{*} \\
(-0.7 \text { to }-0.1)\end{array}$ & $\begin{array}{l}\text { 1980-2018: }-0.14 \\
(-0.3 \text { to } 0.0)\end{array}$ \\
\hline $60-64$ & $\begin{array}{l}\text { 1980-2004: } 3.07 *(2.7 \text { to } 3.4) \\
\text { 2004-2007: } 13.57(-2.1 \text { to } 31.7) \\
\text { 2007-2018: }-0.54(-1.5 \text { to } 0.5)\end{array}$ & $\begin{array}{l}\text { 1980-2018: } 0.11 \\
(-0.1 \text { to } 0.3)\end{array}$ & $\begin{array}{l}\text { 1980-2008: } 3.08^{*} \\
\quad(2.8 \text { to } 3.3) \\
\text { 2008-2018: }-0.6 \mid \\
\quad(-1.6 \text { to } 0.3)\end{array}$ & $\begin{array}{l}|980-200|:-1.09 *(-1.8 \\
\text { to }-0.4) \\
200|-20| 8: 0.85 *(0.0 \text { to } \\
\text { I.7) }\end{array}$ & $\begin{array}{l}\text { I980-1996: } 2.40^{*} \\
\quad(1.8 \text { to } 3.0) \\
\text { 1996-2018: }-0.01 \\
(-0.4 \text { to } 0.4)\end{array}$ & $\begin{array}{l}1980-2018: 0.23^{*}(0.0 \\
\text { to } 0.4)\end{array}$ \\
\hline 65-69 & $\begin{array}{l}\text { 1980-2004: } 3.07^{*}(2.7 \text { to } 3.4) \\
\text { 2004-201 I: } 6.63^{*}(4.1 \text { to } 9.3) \\
\text { 201 I-2018: }-0.04 \text { (-1.9 to } 1.9)\end{array}$ & $\begin{array}{l}\text { 1980-2004: }-0.13 \\
\quad(-0.4 \text { to } 0.1) \\
2004-2018: 0.86^{*} \\
(0.3 \text { to } 1.4)\end{array}$ & $\begin{array}{l}\text { 1980-1993: } 5.85^{*} \\
(4.5 \text { to } 7.2) \\
\text { 1993-2018: } 2.38^{*} \\
\text { (1.9 to } 2.8)\end{array}$ & $\begin{array}{c}\text { 1980-1996: } 0.95(0.0 \text { to } \\
2.0) \\
\text { 1996-2004: }-2.67 \\
(-7.6 \text { to } 2.5) 2004-2018: \\
3.97 *(2.9 \text { to } 5.1)\end{array}$ & $\begin{array}{l}\text { |980-199|: } 4.08^{*} \\
(2.3 \text { to } 5.9) \\
|99|-20 \mid 8: 0.58^{*} \\
(0.2 \text { to } 1.0)\end{array}$ & $\begin{array}{c}\text { 1980-20| 8: } 0.91 *(0.7 \\
\text { to I.2) }\end{array}$ \\
\hline 70-74 & $\begin{array}{l}\text { 1980-1988: I.02 }(-1.5 \text { to } 3.6) \\
\text { 1988-1993: } 7.63^{*}(3.4 \text { to } 12.1) \\
\text { 1993-2006: } 0.91^{*}(0.1 \text { to } 1.7) \\
\text { 2006-2018: 3.08* (2.3 to } 3.9)\end{array}$ & $\begin{array}{l}\text { 1980-1983: }\left.11.4\right|^{*} \\
(3.8 \text { to } 19.5) \\
\text { 1983-2009: }-0.07 \\
(-0.4 \text { to } 0.3) \\
2009-2018: 2.30^{*} \\
(1.0 \text { to } 3.6)\end{array}$ & $\begin{array}{l}\text { 1980-1994: } 6.52^{*} \\
(5.7 \text { to } 7.4) \\
\text { 1994-2018: }\left.3.0\right|^{*} \\
(2.6 \text { to } 3.4)\end{array}$ & $\begin{array}{l}\text { 1980-2007: }-0.15(-0.6 \text { to } \\
0.3) \\
2007-2018: 5.47 * \text { (3.8 to } \\
7.2)\end{array}$ & $\begin{array}{l}\text { 1980-1993: } 4.29 * \\
(3.0 \text { to } 5.6) \\
1993-2018: 0.75^{*} \\
(0.3 \text { to } 1.2)\end{array}$ & $\begin{array}{c}1980-2018: 1.56 *(1.3 \\
\text { to } 1.8)\end{array}$ \\
\hline
\end{tabular}

(Continued) 
Table 2 (Continued).

\begin{tabular}{|c|c|c|c|c|c|c|}
\hline 75-79 & $\begin{array}{l}\text { 1980-1995:3.74* (2.6 to } 4.9) \\
1995-2018: 2.44 *(1.9 \text { to } 3.0)\end{array}$ & $\begin{array}{l}\text { 1980-1994: } 2.09 * \\
(1.6 \text { to } 2.6) \\
\text { 1994-201।: }-0.15 \\
(-0.6 \text { to } 0.3) \\
2011-2018: 2.57^{*} \\
\text { (I.2 to } 4.0)\end{array}$ & $\begin{array}{l}\text { 1980-2018: } 3.99 * \\
(3.7 \text { to } 4.3)\end{array}$ & $\begin{array}{c}\text { 1980-2007: }-0.12(-0.7 \text { to } \\
0.5) \\
2007-2018: 6.85 *(4.7 \text { to } \\
9.1)\end{array}$ & $\begin{array}{l}\text { 1980-1995: } 4.56^{*} \\
(3.6 \text { to } 5.6) \\
1995-2018: 0.77^{*} \\
(0.3 \text { to } 1.3)\end{array}$ & $\begin{array}{c}\text { 1980-2003: } 2.81 *(2.2 \\
\text { to } 3.4) \\
\text { 2003-2018: } 1.33 *(0.4 \\
\text { to } 2.3)\end{array}$ \\
\hline $80-84$ & $\begin{array}{l}1980-2000: 3.43^{*}(2.5 \text { to } 4.3) \\
2000-2007:-0.57(-4.7 \text { to } 3.8) \\
2007-2018: 4.28^{*}(2.5 \text { to } 6.1)\end{array}$ & $\begin{array}{l}\text { 1980-20|4: } 1.12^{*} \\
\quad(0.8 \text { to } 1.4) \\
20|4-20| 8: 6.06 \\
(-0.9 \text { to } 13.6)\end{array}$ & $\begin{array}{l}\text { 1980-2018: } 4.24^{*} \\
(3.8 \text { to } 4.6)\end{array}$ & $\begin{array}{l}\text { I980-2007: } 0.12(-0.6 \text { to } \\
0.8) \\
2007-2018: 8.40 *(5.9 \text { to } \\
10.9)\end{array}$ & $\begin{array}{l}\text { 1980-2002: } 3.54^{*} \\
(2.7 \text { to } 4.3) \\
2002-2018:-0.05 \\
(-1.1 \text { to } 1.0)\end{array}$ & $\begin{array}{c}1980-2018: 2.72^{*}(2.4 \\
\text { to } 3.0)\end{array}$ \\
\hline $85+$ & $\begin{array}{l}\text { 1980-1995: } 3.10 *(1.9 \text { to } 4.3) \\
\text { 1995-2018: } 0.99 *(0.4 \text { to } 1.6)\end{array}$ & $\begin{array}{c}\text { 1980-2005: } 2.76^{*} \\
\text { (2.3 to } 3.3) \\
2005-2013:-0.47 \\
(-3.3 \text { to } 2.5) \\
20 \mid 3-2018: 10.75^{*} \\
\text { (5.5 to } 16.3)\end{array}$ & $\begin{array}{c}\text { 1980-1995: } 4.53^{*} \\
\text { (2.9 to } 6.1) \\
\text { 1995-2004: } 0.64 \\
(-3.4 \text { to } 4.9) \\
2004-2018: 5.07^{*} \\
\text { (3.5 to } 6.7)\end{array}$ & $\begin{array}{c}\text { 1980-2007: } 1.02 \text { (0.0 to } \\
2.1) \\
2007-2018: 11.34 *(7.5 \text { to } \\
\text { 15.3) }\end{array}$ & $\begin{array}{l}\text { 1980-1996: } 5.72 * \\
\text { (3.9 to } 7.6) \\
\text { 1996-2018: }-0.28 \\
(-1.4 \text { to } 0.8)\end{array}$ & $\begin{array}{c}1980-2018: 3.30 *(2.7 \\
\text { to } 3.9)\end{array}$ \\
\hline \multicolumn{7}{|c|}{ B Cervical, Vulvar, and Vaginal } \\
\hline \multirow[t]{2}{*}{ Age, years } & \multicolumn{2}{|c|}{ Cervix Cancer Trends } & \multicolumn{2}{|c|}{ Vulvar Cancer Trends } & \multicolumn{2}{|c|}{ Vaginal Cancer Trends } \\
\hline & $\begin{array}{c}\text { Incidence, APC } \\
(95 \% \mathrm{Cl})\end{array}$ & $\begin{array}{c}\text { Mortality, APC } \\
(95 \% \mathrm{Cl})\end{array}$ & $\begin{array}{c}\text { Incidence, APC } \\
(95 \% \mathrm{CI})\end{array}$ & $\begin{array}{c}\text { Mortality, APC ( } 95 \% \\
\text { Cl) }\end{array}$ & $\begin{array}{c}\text { Incidence, APC } \\
(95 \% \mathrm{Cl})\end{array}$ & $\begin{array}{l}\text { Mortality, APC, } \\
(95 \% \mathrm{Cl})\end{array}$ \\
\hline $0-4$ & - & - & - & - & - & - \\
\hline 5-9 & - & - & - & - & - & - \\
\hline $10-14$ & - & - & - & - & - & - \\
\hline $15-19$ & - & - & - & - & - & - \\
\hline $20-24$ & $\begin{array}{c}\text { 1980-1999: } 0.35(-4.2 \text { to } 5.1) 1999-2003: \\
-29.66(-60.9 \text { to } 26.4) \\
\text { 2003-2018: } 4.45 \text { (-0.6 to } 9.8)\end{array}$ & - & - & - & - & - \\
\hline $25-29$ & 1980-2018: $-2.87^{*}(-3.4$ to -2.3$)$ & $\begin{array}{l}\text { 1980-2015: }-6.01 * \\
(-7.1 \text { to }-5.0) \\
2015-2018: 30.96 \\
(-14.0 \text { to } 99.3)\end{array}$ & - & - & - & - \\
\hline
\end{tabular}




\begin{tabular}{|c|c|c|c|c|c|c|}
\hline 30-34 & 1980-2018: $-2.96 *(-3.4$ to -2.5$)$ & $\begin{array}{c}\text { 1980-1990: }-1.38 \\
(-5.7 \text { to } 3.1) \\
\text { 1990-2018: }-6.03^{*} \\
(-6.8 \text { to }-5.2)\end{array}$ & - & - & - & - \\
\hline $35-39$ & $\begin{array}{l}\text { 1980-1991: I.II (-0.6 to } 2.8) \\
\text { 199|-2018: }-4.19 *(-4.6 \text { to }-3.8)\end{array}$ & $\begin{array}{c}1980-1994:-0.18 \\
\quad(-1.6 \text { to } 1.2) \\
1994-2016:-6.04^{*} \\
(-6.7 \text { to }-5.3) \\
2016-2018: 14.39 \\
(-12.8 \text { to } 50.1)\end{array}$ & $\begin{array}{c}\text { 1999-2018: }-5.30^{*} \\
(-9.3 \text { to }-1.1)\end{array}$ & - & - & - \\
\hline $40-44$ & $\begin{array}{c}\text { 1980-1992: } 2.96^{*}(2.0 \text { to } 3.9) \\
\text { 1992-2007: }-3.7^{*}(-4.3 \text { to } 3.1) \\
\text { 2007-2018: }-5.63^{*}(-6.5 \text { to } 4.8)\end{array}$ & $\begin{array}{l}\text { 1980-1996: } 1.40^{*} \\
(0.2 \text { to } 2.6) \\
1996-20 \mid 8:-5.93^{*} \\
(-6.6 \text { to }-5.2)\end{array}$ & $\begin{array}{l}|999-2018:-5.0|^{*} \\
(-7.9 \text { to }-2.1)\end{array}$ & - & - & - \\
\hline $45-49$ & $\begin{array}{c}\text { 1980-2000: } 0.92 *(0.2 \text { to } 1.7) \\
2000-2018:-5.57^{*}(-6.2 \text { to }-4.9)\end{array}$ & $\begin{array}{c}\text { 1980-2004: }-0.58^{*} \\
(-1.1 \text { to }-0.0) \\
2004-2018: \\
-6.10^{*}(-7.1 \text { to }-5.1)\end{array}$ & $\begin{array}{c}\text { 1999-2016: } \\
-1.42(-3.6 \text { to } 0.8) \\
2016-2018: \\
-43.12(-69.8 \text { to } 7.0)\end{array}$ & - & - & - \\
\hline $50-54$ & $\begin{array}{l}\text { 1980-2008: }-0.46^{*}(-0.9 \text { to } 0.0) \\
\text { 2008-2018: }-6.85^{*}(-8.5 \text { to }-5.1)\end{array}$ & $\begin{array}{l}\text { 1980-2009: }-0.38 \\
\quad(-0.8 \text { to } 0.0) \\
2009-2018:-6.95^{*} \\
(-9.0 \text { to }-4.9)\end{array}$ & $\begin{array}{l}\text { 1999-2018: }-1.26 \\
\quad(-2.8 \text { to } 0.3)\end{array}$ & $\begin{array}{c}\text { 1999-2015: } 2.56(-0.8 \text { to } \\
6.1) \\
\text { 2015-2018: }-32.25(-56.4 \\
\text { to } 5.3)\end{array}$ & $\begin{array}{l}|999-2018:-3.9| * \\
(-7.2 \text { to }-0.5)\end{array}$ & $\begin{array}{l}\text { |999-20|8: }-4.85 * \\
\quad(-9.5 \text { to } 0.0)\end{array}$ \\
\hline $55-59$ & $\begin{array}{l}\text { 1980-1993: }-2.61 *(-3.5 \text { to }-1.7) \\
\text { 1993-2010: } 0.06(-0.6 \text { to } 0.7) \\
2010-2018:-6.65 *(-8.3 \text { to }-5.0)\end{array}$ & $\begin{array}{l}\text { I980-200I: }-2.48^{*} \\
(-3.1 \text { to }-1.8) \\
200 \mid-2007: 3.96 \\
(-1.1 \text { to } 9.2) \\
2007-2018:-4.16^{*} \\
(-5.6 \text { to }-2.7)\end{array}$ & $\begin{array}{l}\text { 1999-2018: } 0.35 \\
(-1.6 \text { to } 2.3)\end{array}$ & $\begin{array}{c}\text { 1999-20।8: } 2.37(-1.0 \text { to } \\
5.8)\end{array}$ & $\begin{array}{l}\text { 1999-2018: } 1.38 \\
(-1.9 \text { to } 4.8)\end{array}$ & $\begin{array}{l}\text { 1999-2018: }-2.89 \\
\quad(-7.8 \text { to } 2.3)\end{array}$ \\
\hline $60-64$ & $\begin{array}{l}\text { 1980-2004: }-2.40 *(-2.8 \text { to }-2.0) \\
\text { 2004-2007: } 5.51(-9.1 \text { to } 22.4) \\
\text { 2007-2018: }-2.49 *(-3.5 \text { to }-1.5)\end{array}$ & $\begin{array}{l}\text { 1980-2005: }-2.17^{*} \\
(-2.7 \text { to }-1.6) \\
2005-2018:-0.17 \\
(-1.4 \text { to } 1.1)\end{array}$ & $\begin{array}{l}\text { 1999-2018: } 1.28 \\
\text { (-0.1 to } 2.7)\end{array}$ & $\begin{array}{c}\text { 1999-20 |8: } 0.54(-1.5 \text { to } \\
2.6)\end{array}$ & $\begin{array}{l}\text { 1999-2018: } 0.99 \\
\quad(-1.6 \text { to } 3.6)\end{array}$ & - \\
\hline
\end{tabular}


Table 2 (Continued).

\begin{tabular}{|c|c|c|c|c|c|c|}
\hline 65-69 & $\begin{array}{l}\text { 1980-1985: } 4.72(-1.6 \text { to II.4) } \\
\text { 1985-2006: }-2.99 *(-3.6 \text { to }-2.4) \\
\text { 2006-2018: } 0.04(-1.1 \text { to } 1.2)\end{array}$ & $\begin{array}{c}\text { 1980-1988: } 1.60 \\
(-2.2 \text { to } 5.6) \\
\text { 1988-2001: }-3.93^{*} \\
(-5.1 \text { to }-2.7) \\
200 \mid-2018:-0.52 \\
(-1.2 \text { to } 0.2)\end{array}$ & $\begin{array}{l}\text { 1999-2018: } 0.30 \\
(-0.8 \text { to } 1.4)\end{array}$ & $\begin{array}{c}\text { 1999-2018: }-0.29(-2.5 \text { to } \\
\text { ।.9) }\end{array}$ & $\begin{array}{l}\text { 1999-2014: }-3.18^{*} \\
(-5.9 \text { to }-0.4) \\
2014-2018: 18.29 \\
(-4.4 \text { to } 46.4)\end{array}$ & - \\
\hline 70-74 & $\begin{array}{c}\text { 1980-1995: }-0.20(-0.9 \text { to } 0.5) \\
\text { 1995-2005: }-4.86^{*}(-6.3 \text { to }-3.4) \\
2005-2018 \text { : } \\
-0.53(-1.3 \text { to } 0.2)\end{array}$ & $\begin{array}{c}\text { 1980-1995: } \\
-0.75 \text { (-1.7 to } 0.3) \\
1995-2008:-4.08^{*} \\
(-5.5 \text { to }-2.7) \\
2008-2018: \\
0.92 \text { (-0.7 to } 2.6)\end{array}$ & $\begin{array}{l}\text { 1999-2018: } 0.73^{*} \\
\quad(0.1 \text { to } 1.4)\end{array}$ & $\begin{array}{c}\text { 1999-2018: } 0.26(-1.2 \text { to } \\
\text { ।.8) }\end{array}$ & $\begin{array}{l}\text { 1999-2018: } 1.04 \\
(-1.5 \text { to } 3.7)\end{array}$ & $\begin{array}{c}\text { |999-20|8: }-4.14^{*} \\
(-7.3 \text { to }-0.9)\end{array}$ \\
\hline $75-79$ & $\begin{array}{c}\text { 1980-1994: } 0.29(-1.0 \text { to } 1.6) \\
\text { 1994-2018: }-3.02 *(-3.6 \text { to }-2.5)\end{array}$ & $\begin{array}{l}\text { 1980-1999: }-0.21 \\
\quad(-0.9 \text { to } 0.5) \\
\text { 1999-2002: }-8.60 \\
(-24.0 \text { to } 9.9) \\
2002-2018:-1.85^{*} \\
(-2.5 \text { to }-1.2)\end{array}$ & $\begin{array}{l}\text { 1999-2018: } 0.73 \\
(-0.1 \text { to } 1.5)\end{array}$ & $\begin{array}{c}\text { 1999-2018: } 0.50(-0.8 \text { to } \\
\text { ।.8) }\end{array}$ & $\begin{array}{l}\text { 1999-2018: }-1.59 \\
(-4.3 \text { to } 1.2)\end{array}$ & $\begin{array}{l}\text { 1999-20|8: }-2.73 \\
(-5.5 \text { to } 0.1)\end{array}$ \\
\hline $85+$ & 1980-2018: $-1.23^{*}(-2.0$ to -0.4$)$ & $\begin{array}{c}1980-2018: \\
0.46(0.0 \text { to } 0.9)\end{array}$ & $\begin{array}{l}\text { |999-2018: }\left.1.7\right|^{*} \\
\text { (0.6 to } 2.8)\end{array}$ & $\begin{array}{c}\text { 1999-2018: } 2.64 *(1.3 \text { to } \\
4.0)\end{array}$ & $\begin{array}{l}1999-2018: 1.21 \\
(-2.2 \text { to } 4.8)\end{array}$ & $\begin{array}{c}\text { 1999-2018: }-0.01 \\
(-2.9 \text { to } 3.0)\end{array}$ \\
\hline
\end{tabular}

Notes: *Statistically significant. Missing data for 1981, 1985-1986, and 1997-1998. No data on vulvar and vaginal cancers before 1999 are available because of the lack of separate codes for them in the International Classification of Diseases, 9th revision.

Abbreviation: APC - annual percentage change; $\mathrm{Cl}$ - confidence interval. 
States, increases in both incidence and mortality rates of CUC were noted in 1999-2015, with the differences in incidence mostly resulting from increases in endometrial cancer. ${ }^{38}$

The introduction of screening for $\mathrm{CC}$ proved to be a turning point in $\mathrm{CC}$ prevention worldwide, leading to striking decreases in the prevalence of $\mathrm{CC}$ in those countries in which Pap smears are generally available. ${ }^{39}$ Widespread use of secondary prophylaxis of CC in Poland, which includes Pap smear, HPV testing, and colposcopy, together with an implementation of vaccinations against HPV and an improvement in socioeconomic status, are most likely the reason for declining trends in the incidence and mortality of $\mathrm{CC}$ that have begun and are now present in all age groups combined as well as in 5-year age groups. In the first decade of the $21^{\text {st }}$ century, stabilization of trends in CC incidence were reported in Norway and in the United States, presumably because the upper limits of the potential of existing screening program aimed at decreasing incidence rates were reached. ${ }^{40,41}$ In Poland, structured, nationwide CC screening programs adhering to European guidelines were launched in 2007, and, to some degree, may have accelerated reductions in incidence and mortality observed in the 50-54 years and 55-59 years age groups. The incidence and mortality of VUC and VAC in younger women may have been reduced by $\mathrm{CC}$ screening to some extent as well.

Early age at first intercourse and multiple sexual partners are proven risk factors for the development of CC, VUC, and VAC. ${ }^{42-44}$ Age at the onset of sexual activity has decreased significantly in the past 50 years in Poland. Populationbased studies point to a decrease in the mean age at onset of sexual activity by 1 or even 2.5 years among women born in the 1990s or early 2000s compared with women born during the 1960s and 1970s. ${ }^{21,45}$ The average number of sexual partners is also presumed to have increased in recent times. For these reasons, increasing trends in the incidence and mortality of these cancers, especially among younger women, may be expected. Results from our study appear contrary to those assumptions.

Because VAC is rare and thus prone to inconsistencies in incidence among consecutive years, it is hard to detect significant trends. Nevertheless, the generally decreasing trends in the incidence of VAC observed in our study are similar to results from some other countries. In the United States and Japan, a statistically significant average annual decrease in the incidence of VAC has been reported, whereas no such trends have been noted in England and Norway. ${ }^{40,46-48}$ Improving socioeconomic status, accessible gynecological care, and advancements in assessment and management may have been responsible for the observed reductions in the incidence and mortality of primary VAC, which was most remarkable in the 50-54 years age group, and for the reduced trends in the incidence of VUC among younger women. However, increasing trends in VUC incidence in older women may reflect previous underdiagnosis in this population. Moreover, because advanced age is more likely to be associated with a diagnosis of late-stage VUC, increasing incidence in older age groups may help explain the trends of increasing mortality found in our study among the oldest and the allages groups. Trends in VUC incidence in Poland stand in stark contrast to trends observed in other countries, such as in Germany, England, Norway, and the Netherlands, where increasing trends in incidence have been reported, especially in women younger than age 60 years. ${ }^{49-52}$ Increasing incidence rates in younger women have also been observed in Denmark and Australia, ${ }^{53,54}$ whereas a decline in the incidence rate in all ages in Italy has been noted; the change in Italian rates is attributed mostly to decreasing incidence among older women. ${ }^{55}$ Diminishing popularity of cigarette smoking during 1995-2015 may also have contributed to the reductions in the incidence and mortality of VUC, VAC, and CC among younger women, because being a current smoker is associated with a higher HPV baseline load and is a risk factor for HPV-related squamous cell carcinoma development. ${ }^{42,56-58}$

The 1980-2018 period was associated with significant changes in gynecological cancer and BC incidence and mortality. The observed tendencies raise concerns, especially because the trends are usually worse compared with those in other European countries, as discussed earlier in this section. Furthermore, the recent SARS-CoV-2 pandemic may have a negative impact on mortality tendencies observed in the next few years because of postponed screening and diagnostic processes and treatment delays. ${ }^{59}$ Moreover, continually increasing rates of CUC incidence and mortality and trends of increased $\mathrm{BC}$ incidence might be expected as a result of observed socioeconomic changes, predicted increases in obesity prevalence, and the exhaustion or lack of viable screening methods. ${ }^{60}$ Nevertheless, increasing awareness, growing recognition of screening importance, and the introduction of new accessible therapeutic options may eventually lead to more favorable tendencies in the future. Finally, the possible introduction of a long-awaited national anti-HPV 
vaccination program would carry great potential for reducing CC, VUC, and VAC incidence and mortality rates in the future, as shown in Norwegian and Australian analyses. ${ }^{40,61}$

The strength of our study lies in the volume of data available for analysis. The population data were reliably collected, ensuring that the results of this study are valid for the entire Polish population. The age-standardized rates were calculated using the world standard population as a reference, thus allowing use of the results to compare trends in Poland with those in other countries. This study also has some limitations. First, the registry of data did not reveal the causes for changes in documentation. Second, this study does not take into consideration specific histological types, the incidence of which varies depending on the age of the patient. Moreover, the statistical analyses performed in the Joinpoint Regression Program do not include the use of Bayesian methods.

\section{Conclusion}

The results of this study showed significant increases in the incidences of OC, CUC, and BC and a decrease in the incidence of CC and VAC. The incidence trends for VUC were stable Mortality trends for BC fluctuated during the study period; since 2010, BC mortality has begun to increase. Throughout the study period, mortality from VUC and CUC increased, whereas mortality from CC decreased. Mortality from OC was stable and not significant. Furthermore, analysis of the data showed a correlation between age groups and rates of incidence and mortality of each cancer, even among very rare malignancies, such as VUC and VAC. The observed trends need additional analysis.

\section{Data Sharing Statement}

Data from the Polish National Cancer Registry are publicly available.

\section{Ethics Approval}

Not required since publication of data from the Polish National Cancer Registry is permitted generally.

\section{Disclosure}

The authors report no conflicts of interest in this work.

\section{References}

1. Sung H, Ferlay J, Siegel RL, et al. Global cancer statistics 2020: GLOBOCAN estimates of incidence and mortality worldwide for 36 cancers in 185 countries. CA a Cancer J Clin. 2021;71(3):209-249. doi:10.3322/caac.21660

2. Brończyk-Puzoń A, Koszowska A, Nowak J, Dittfeld A, Bieniek J. Epidemiologia otyłości na świecie i w Polsce. [Epidemiology of obesity in the world and in Poland]. Forum Zaburzen Metabolicznych. 2014;5(1):1-5. Polish.

3. Centrala Narodowego Funduszu Zdrowia, Departament Analiz i Strategii. [Headquarters of the National Health Fund, Department of Analysis and Strategy]. [Homepage on the Internet]. Cukier, otyłość - konsekwencje. Przegląd literatury, szacunki dla Polski. [Sugar, obesity - consequences. Literature review, estimates for Poland]. 2019. Available from: https://pacjent.gov.pl/artykul/cukrzyca-w-liczbach/. Accessed March $29,2021$. Polish.

4. Momenimovahed Z, Salehiniya H. Epidemiological characteristics of and risk factors for breast cancer in the world. BCTT. 2019;11:151-164. doi: $10.2147 /$ bctt.s176070

5. Kamińska M, Ciszewski T, Łopacka-szatan K, Miotła P, Starosławska E. Breast cancer risk factors. Menopause Review Przeglad Menopauzalny. 2015;14(3):196-202. doi:10.5114/pm.2015.54346

6. Nindrea RD, Aryandono T, Lazuardi L. Breast cancer risk from modifiable and non-modifiable risk factors among women in southeast asia: a metaanalysis. Asian Pac J Cancer Prev. 2017;18(12). doi:10.22034/APJCP.2017.18.12.3201

7. Ferlay J, Colombet M, Soerjomataram I, et al. Cancer statistics for the year 2020: an overview. Int J Cancer. 2021;149(4):778-789. doi:10.1002/ ijc. 33588

8. Kashyap N, Krishnan N, Kaur S, Ghai S. Risk factors of cervical cancer: a case-control study. Asia-Pacific Journal of Oncology Nursing. 2019;6 (3):308. doi:10.4103/apjon.apjon_73_18

9. Zhang S, Xu H, Zhang L, Qiao Y. Cervical cancer: epidemiology, risk factors and screening. Chin J Cancer. 2020;32(6):720-728. doi:10.21147/j. issn.1000-9604.2020.06.05

10. Lortet-Tieulent J, Ferlay J, Bray F, Jemal A. International patterns and trends in endometrial cancer incidence, 1978-2013. JNCI. 2017;110(4):354361. doi:10.1093/jnci/djx214

11. Ali AT. Risk factors for endometrial cancer. Ceska Gynekologie. 2013;78(5):548.

12. Wentzensen N, Poole EM, Trabert B, et al. Ovarian cancer risk factors by histologic subtype: an analysis from the ovarian cancer cohort consortium. JCO. 2016;34(24):2888-2898. doi:10.1200/jco.2016.66.8178

13. Momenimovahed Z, Tiznobaik A, Taheri S, Salehiniya H. Ovarian cancer in the world: epidemiology and risk factors. IJWH. 2019;11:287-299. doi:10.2147/ijwh.s197604 
14. Duong TH, Flowers LC. Vulvo-Vaginal Cancers: risks, Evaluation, Prevention and Early Detection. Obstet Gynecol Clin North Am. 2007;34 (4):783-802. doi:10.1016/j.ogc.2007.10.003

15. Krajowy Rejestr Nowotworów, Narodowy Instytut Onkologii im. Marii Skłodowskiej-Curie - Państwowy Instytut Badawczy. [Polish National Cancer Registry]. [Homepage on the Internet]. Wojciechowska U, Didkowska J. Zachorowania i zgony na nowotwory złośliwe w Polsce. [Cases and deaths from malignant neoplasms in Poland]. Available from: http://onkologia.org.pl/. Accessed March 29, 2021. Polish.

16. Centers for Disease Control and Prevention. National Center for Health Statistics. [Homepage on the Internet]. Classification of Diseases, Functioning, and Disability. Available from: https:/www.cdc.gov/nchs/icd/index.htm/. Accessed March 29, 2021.

17. Joinpoint Trend Analysis Software. [Homepage on the Internet]. Available from: https://surveillance.cancer.gov/joinpoint/. Accessed March 29, 2021.

18. Clegg LX, Hankey BF, Tiwari R, Feuer EJ, Edwards BK. Estimating average annual per cent change in trend analysis. Statist Med. 2009;28 (29):3670-3682. doi:10.1002/sim.3733

19. National Cancer Institute. [Homepage on the Internet]. Methodology for characterizing trends, cancer trends progress report. Available from: https://progressreport.cancer.gov/methodology/. Accessed October 8, 2021.

20. Polish National Statistical Office. [Homepage on the Internet]. Dzietność kobiet w latach 1960-2019. [Women's fertility in 1960-2019.]. Available from: https://stat.gov.pl/obszary-tematyczne/ludnosc/ludnosc/struktura-ludnosci,16,1.html/. Accessed April 13, 2021.

21. Durda-Masny M, Szwed A, Litwiniuk Z, et al. Time intervals between the age at menarche and the onset of sexual activity in Polish women born between 1959 and 2001. Am J Hum Biol. 2020;33(1). doi:10.1002/ajhb.23436

22. Raglan O, Kalliala I, Markozannes G, et al. Risk factors for endometrial cancer: an umbrella review of the literature. Int J Cancer. 2019;145 (7):1719-1730. doi:10.1002/ijc.31961

23. Menon U, Karpinskyj C, Gentry-Maharaj A. Ovarian Cancer Prevention and Screening. Obstet Gynecol. 2018;131(5):909-927. doi:10.1097/ aog.0000000000002580

24. Boussios S, Moschetta M, Tatsi K, Tsiouris AK, Pavlidis N. A review on pregnancy complicated by ovarian epithelial and non-epithelial malignant tumors: diagnostic and therapeutic perspectives. $J$ Adv Res. 2018;12:1-9. doi:10.1016/j.jare.2018.02.006

25. Ng M, Fleming T, Robinson M, et al. Global, regional, and national prevalence of overweight and obesity in children and adults during 1980-2013: a systematic analysis for the Global Burden of Disease Study 2013. The Lancet. 2014;384(9945):766-781. doi:10.1016/s0140-6736(14)60460-8

26. Didkowska J, Wojciechowska U. Breast cancer in Poland and Europe - Population and statistics Introduction. Nowotwory. 2013;63(2):154.

27. Serwis Rzeczypospolitej Polskiej - Ministerstwo Zdrowia [Polish Ministry of Health]. [Homepage on the Internet]. Programy lekowe - choroby onkologiczne. [Drug programs - oncological diseases]. Available from: https://www.gov.pl/web/zdrowie/choroby-onkologiczne/. Accessed October 9, 2021.

28. Boussios S, Moschetta M, Zarkavelis G, Papadaki A, Kefas A, Tatsi K. Ovarian sex-cord stromal tumours and small cell tumours: pathological, genetic and management aspects. Crit Rev Oncol Hematol. 2017;120:43-51. doi:10.1016/j.critrevonc.2017.10.007

29. Torre LA, Trabert B, DeSantis CE, et al. Ovarian cancer statistics, 2018. CA Cancer J Clin. 2018;68(4):284-296. doi:10.3322/caac.21456

30. Czerw A, Banas T, Juszczyk G, Pitynski K, Nieweglowska D, Ludwin A. Incidence and mortality rates in breast, corpus uteri, and ovarian cancers in Poland (1980-2013): an analysis of population-based data in relation to socio-economic changes. OTT. 2016;9:5521-5530. doi:10.2147/ott. s112187

31. Berkemeyer S, Lemke D, Hense HW. Incidence and mortality trends in German women with breast cancer using age, period and cohort 1999 to 2008. PLoS One. 2016;11(3):e0150723. doi:10.1371/journal.pone.0150723

32. Kvåle R, Myklebust TA, Engholm G, Heinävaara S, Wist E, Møller B. Prostate and breast cancer in four Nordic countries: a comparison of incidence and mortality trends across countries and age groups 1975-2013. Int J Cancer. 2017;141(11):2228-2242. doi:10.1002/ijc.30924

33. Barchuk A, Bespalov A, Huhtala H, et al. Breast and cervical cancer incidence and mortality trends in Russia 1980-2013. Cancer Epidemiol. 2018;55:73-80. doi:10.1016/j.canep.2018.05.008

34. Wojtyla C, Bertuccio P, Wojtyla A, La Vecchia C. European trends in breast cancer mortality, 1980-2017 and predictions to 2025. Eur J Cancer. 2021;152:4-17. doi:10.1016/j.ejca.2021.04.026

35. Carioli G, Malvezzi M, Rodriguez T, Bertuccio P, Negri E, La Vecchia C. Trends and predictions to 2020 in breast cancer mortality in Europe. The Breast. 2017;36:89-95. doi:10.1016/j.breast.2017.06.003

36. Malvezzi M, Carioli G, Rodriguez T, Negri E, La Vecchia C. Global trends and predictions in ovarian cancer mortality. Annals of Oncology. 2016;27(11):2017-2025. doi:10.1093/annonc/mdw306

37. Khazaei Z, Goodarzi E, Sohrabivafa M, Naemi H, Mansori K. Association between the incidence and mortality rates for corpus uteri cancer and human development index (HDI): a global ecological study. Obstet Gynecol Sci. 2020;63(2):141-149. doi:10.5468/ogs.2020.63.2.141

38. Henley SJ, Miller JW, Dowling NF, Benard VB, Richardson LC. Uterine Cancer Incidence and Mortality — United States, 1999-2016. MMWR Morb Mortal Wkly Rep. 2018;67(48):1333-1338. doi:10.15585/mmwr.mm6748a1

39. Safaeian M, Solomon D, Castle PE. Cervical Cancer Prevention-Cervical Screening: science in Evolution. Obstet Gynecol Clin North Am. 2007;34(4):739-760. doi:10.1016/j.ogc.2007.09.004

40. Hansen BT, Campbell S, Nygård M. Long-term incidence trends of HPV-related cancers, and cases preventable by HPV vaccination: a registrybased study in Norway. BMJ Open. 2018;8(2):e019005. doi:10.1136/bmjopen-2017-019005

41. National Cancer Institute. [Homepage on the Internet]. SEER*Explorer: an interactive website for SEER cancer statistics Surveillance Research Program. Available from: https://seer.cancer.gov/explorer/. Accessed April 15, 2021.

42. Berrington De González A, Green J. Comparison of risk factors for invasive squamous cell carcinoma and adenocarcinoma of the cervix: collaborative reanalysis of individual data on 8097 women with squamous cell carcinoma and 1374 women with adenocarcinoma from 12 epidemiological studies. Int J Cancer. 2006;120(4):885-891. doi:10.1002/ijc.22357

43. Brinton L, Nasca P, Mallin K, Baptiste M, Wilbanks G, Richart R. Case-control study of cancer of the vulva. Int J Gynecol Obstet. 1990;33(4):381. doi:10.1016/0020-7292(90)90543-t

44. Daling JR, Madeleine MM, Schwartz SM, et al. A population-based study of squamous cell vaginal cancer: HPV and Cofactors. Gynecol Oncol. 2002;84(2):263-270. doi:10.1006/gyno.2001.6502

45. Durda-Masny M, Jarząbek-Bielecka G, Szwed A, Hanć T, Czapla Z, Kaczmarek M. Trends over time in age at sexual debut among Polish women and underlying socio-economic determinants. Anthr Anz. 2018;75(3):185-191. doi:10.1127/anthranz/2018/0853 
46. Kurdgelashvili G, Dores GM, Srour SA, Chaturvedi AK, Huycke MM, Devesa SS. Incidence of potentially human papillomavirus-related neoplasms in the United States, 1978 to 2007. Cancer. 2013;119(12):2291-2299. doi:10.1002/cncr.27989

47. Yagi A, Ueda Y, Kakuda M, et al. Descriptive epidemiological study of vaginal cancer using data from the Osaka Japan population-based cancer registry. Medicine. 2017;96(32):e7751. doi:10.1097/md.0000000000007751

48. Robinson D, Coupland V, Møller H. An analysis of temporal and generational trends in the incidence of anal and other HPV-related cancers in Southeast England. Br J Cancer. 2009;100(3):527-531. doi:10.1038/sj.bjc.6604871

49. Buttmann-Schweiger N, Klug SJ, Luyten A, et al. Incidence patterns and temporal trends of invasive nonmelanotic vulvar tumors in Germany 1999-2011. A population-based cancer registry analysis. PLoS One. 2015;10(5). doi:10.1371/journal.pone.0128073

50. Lai J, Elleray R, Nordin A, et al. Vulval cancer incidence, mortality and survival in England: age-related trends. BJOG. 2013;121(6):728-738. doi:10.1111/1471-0528.12459

51. Meltzer-Gunnes CJ, Småstuen MC, Kristensen GB, Tropé CG, Lie AK, Vistad I. Vulvar carcinoma in Norway: a 50-year perspective on trends in incidence, treatment and survival. Gynecol Oncol. 2017;145(3):543-548. doi:10.1016/j.ygyno.2017.03.008

52. Schuurman MS, van den Einden LCG, Massuger LFAG, Kiemeney LA, van der Aa MA, de Hullu JA. Trends in incidence and survival of Dutch women with vulvar squamous cell carcinoma. Eur J Cancer. 2013;49(18):3872-3880. doi:10.1016/j.ejca.2013.08.003

53. Baandrup L, Varbo A, Munk C, Johansen C, Frisch M, Kjaer SK. In situ and invasive squamous cell carcinoma of the vulva in Denmark 1978-2007 -a nationwide population-based study. Gynecol Oncol. 2011;122(1):45-49. doi:10.1016/j.ygyno.2011.03.016

54. Barlow EL, Kang Y-J, Hacker NF, Canfell K. Changing Trends in Vulvar Cancer Incidence and Mortality Rates in Australia Since 1982. Int J Gynecol Cancer. 2015;25(9):1683-1689. doi:10.1097/igc.0000000000000547

55. Mancini S, Bucchi L, Baldacchini F, et al. Incidence trends of vulvar squamous cell carcinoma in Italy from 1990 to 2015 . Gynecol Oncol. 2020;157(3):656-663. doi:10.1016/j.ygyno.2020.03.013

56. Xi LF, Koutsky LA, Castle PE, et al. Relationship Between Cigarette Smoking and Human Papilloma Virus Types 16 and 18 DNA Load. Cancer Epidemiol Biomarkers Prev. 2009;18(12):3490-3496. doi:10.1158/1055-9965.epi-09-0763

57. Madsen BS, Jensen HL, van den Brule AJC, Wohlfahrt J, Frisch M. Risk factors for invasive squamous cell carcinoma of the vulva and vaginaPopulation-based case-control study in Denmark. Int J Cancer. 2008;122(12):2827-2834. doi:10.1002/ijc.23446

58. Dąbrowska K, Sierosławski J, Wieczorek Ł. Trends in tobacco-related behaviour among young people in Poland from 1995 to 2015 against a background of selected European countries. Alcoholism Drug Addiction. 2018;31(1):65-82. doi:10.5114/ain.2018.78816

59. Li Y, Wang X, Wang W. The Impact of COVID-19 on Cancer. Infect Drug Resist. 2021;14:3809-3816. doi:10.2147/IDR.S324569

60. Pineda E, Sanchez-Romero LM, Brown M, et al. Forecasting Future Trends in Obesity across Europe: the Value of Improving Surveillance. Obes Facts. 2018;11(5):360-371. doi:10.1159/000492115

61. Patel C, Brotherton JM, Pillsbury A, et al. The impact of 10 years of human papillomavirus (HPV) vaccination in Australia: what additional disease burden will a nonavalent vaccine prevent? Euro Surveill. 2018;23(41):1700737. doi:10.2807/1560-7917. ES.2018.23.41.1700737

\section{Publish your work in this journal}

Clinical Epidemiology is an international, peer-reviewed, open access, online journal focusing on disease and drug epidemiology, identification of risk factors and screening procedures to develop optimal preventative initiatives and programs. Specific topics include: diagnosis, prognosis, treatment, screening, prevention, risk factor modification, systematic reviews, risk \& safety of medical interventions, epidemiology \& biostatistical methods, and evaluation of guidelines, translational medicine, health policies \& economic evaluations. The manuscript management system is completely online and includes a very quick and fair peer-review system, which is all easy to use.

Submit your manuscript here: https://www.dovepress.com/clinical-epidemiology-journal 